\title{
Occipital Nerve Field Transcranial Direct Current Stimulation Normalizes Imbalance Between Pain Detecting and Pain Inhibitory Pathways in Fibromyalgia
}

\author{
Dirk De Ridder ${ }^{1,2} \cdot$ Sven Vanneste Vi,2,3 $^{1,2}$
}

Published online: 21 December 2016

(C) The American Society for Experimental NeuroTherapeutics, Inc. 2016

\begin{abstract}
Occipital nerve field (OCF) stimulation with subcutaneously implanted electrodes is used to treat headaches, more generalized pain, and even failed back surgery syndrome via unknown mechanisms. Transcranial direct current stimulation (tDCS) can predict the efficacy of implanted electrodes. The purpose of this study is to unravel the neural mechanisms involved in global pain suppression, mediated by occipital nerve field stimulation, within the realm of fibromyalgia. Nineteen patients with fibromyalgia underwent a placebocontrolled OCF tDCS. Electroencephalograms were recorded at baseline after active and sham stimulation. In comparison with healthy controls, patients with fibromyalgia demonstrate increased dorsal anterior cingulate cortex, increased premotor/ dorsolateral prefrontal cortex activity, and an imbalance between pain-detecting dorsal anterior cingulate cortex and painsuppressing pregenual anterior cingulate cortex activity, which is normalized after active tDCS but not sham stimulation associated with increased pregenual anterior cingulate cortex activation. The imbalance improvement between the pregenual anterior cingulate cortex and the dorsal anterior cingulate cortex is related to clinical changes. An imbalance assumes these areas communicate and, indeed, abnormal functional connectivity between the dorsal anterior cingulate cortex and pregenual anterior cingulate cortex is noted to be caused by a dysfunctional effective connectivity from the
\end{abstract}

Sven Vanneste

sven.vanneste@utdallas.edu

1 Department of Surgical Sciences, Dunedin School of Medicine, University of Otago, Dunedin, New Zealand

2 BRAI2N, Sint Augustinus Hospital Antwerp, Antwerp, Belgium

3 School of Behavioral and Brain Sciences, The University of Texas at Dallas, Dallas, TX, USA pregenual anterior cingulate cortex to the dorsal anterior cingulate cortex, which improves and normalizes after real tDCS but not sham tDCS. In conclusion, OCF tDCS exerts its effect via activation of the descending pain inhibitory pathway and de-activation of the salience network, both of which are abnormal in fibromyalgia.

Keywords Fibromyalgia $\cdot$ tDCS $\cdot$ Antinociceptive $\cdot$ Salience $\cdot$ Pain $\cdot$ Inhibitory $\cdot \mathrm{C} 2 \cdot$ Occipital nerve $\cdot$ Connectivity $\cdot$ Balance

\section{Introduction}

Occipital nerve field stimulation with subcutaneously implanted electrodes is used to treat primary headache syndromes [1], more generalized pain such as fibromyalgia [2, 3], and even failed back surgery syndrome [4]. Transcranial direct current stimulation (tDCS) can predict the efficacy of implanted electrodes [5], suggesting that both techniques might exert a similar effect on pain processing in the brain. How electrical stimulation with tDCS or implanted electrodes exerts its elusive effect on global pain perception is not well understood, though hypotheses have been proposed [2]. A positron emission tomography (PET) scan study in patients with cluster headache showed that the pregenual anterior cingulate cortex, a major component of the descending pain inhibitory system [6, 7], is involved in the pain-suppressing effect of occipital (i.e., C2) nerve field stimulation [8]. Development and sustenance of chronic pain has been linked to functional [9] and structural [10] connectivity changes between the reward system (ventral striatum) and the pregenual anterior cingulate part of the descending pain inhibitory antinociceptive system. 
Fibromyalgia is a disorder characterized by chronic complaints of spontaneous widespread pain in all 4 quadrants of the body and is accompanied by fatigue, poor sleep, distress, depression, and/or cognitive dysfunction that is not relieved by rest [11]. Most of the fibromyalgia-associated symptoms can be attributed to categories of hypersensitivity, including somatosensory hypersensitivity (generalized pain, headaches, jaw tightness, morning stiffness, paresthesia), hypersensitivity to other senses (sound, odor, chemical), hypersensitivity in the autonomic nervous system (irritable bowel, urinary urgency, dryness of mouth and eyes, cold swollen hands), and emotional hypersensitivity (anxiety, depression) [12].

The exact mechanism underlying this seemingly complex symptomatology is not known, but it has been proposed that an increased facilitatory modulation contributing to sensitization of the central nervous system and a dysfunctional descending pain inhibitory antinociceptive pathway drive the pain symptoms of fibromyalgia [13]. Functional and structural neuroimaging studies have provided evidence for this existing hypothesis demonstrating structural activity, functional activity, and connectivity changes, resulting in enhanced pain facilitation in combination with defective inhibition of nociceptive signals, which augments pain perception [14]. Activity changes have been found in the insula, the anterior cingulate cortex, and prefrontal cortex, which are all areas that represent a general salience network, thus signifying behaviorally relevant stimuli processing $[15,16]$. These brain activity changes in fibromyalgia are similar to what has been found in patients with chronic back pain, which was interpreted as a lasting effect of pain on brain function [17]. In addition, a deficient pain-inhibitory pathway starting from the pregenual anterior cingulate cortex has also been found in patients with fibromyalgia [14]. The question arises whether the general hypersensitivity and pain in fibromyalgia results predominantly from increased activity in the pain-sensitizing medial pain pathway (also known as a nonspecific salience system) [15, 18, 19], a decrease in the descending pain inhibitory antinociceptive pathway [14], or whether it should be considered more as a balance problem between pain input and pain inhibition [20]. Furthermore, the question arises whether neuromodulation can influence the pathophysiological mechanism underlying fibromyalgia symptoms and its related pain.

Recently, it was demonstrated that direct stimulation of the occipital nerve field via subcutaneously implanted electrodes in patients with fibromyalgia exerts an overall beneficial effect on pain symptoms, as well as quality of life, fatigue, and sleep quality $[3,21,22]$. Furthermore, tDCS can be used to modulate noninvasively the occipital nerve field producing similar results to the implants $[5,23]$, and can predict the results of subsequent subcutaneous occipital nerve field stimulation via implanted electrodes [5]. Occipital nerve field stimulation has a direct impact on the latencies of the laser evoked potentials in patients with fibromyalgia, confirming that this type stimulation alters the processing of the painful stimulus at a central level [24]. During occipital nerve field stimulation, functional magnetic resonance imaging and PET data have demonstrated that activity in the pregenual and dorsal anterior cingulate cortex, insula, somatosensory cortex, and ventroposterolateral nuclei of the thalamus are effectively modulated in healthy subjects $[8,25]$. Interestingly, these structures are involved in attention to pain, pain intensity, and the emotional component of pain perception [26].

The current study aims to disentangle how occipital nerve field stimulation exerts its pain inhibitory effect, as well as whether the pain in fibromyalgia is due to an imbalance between pain provoking pathways (contributing to pain sensitization) and descending pain-inhibitory pathways. This is investigated by using source-localized resting-state electroencephalogram (EEG) activity and connectivity analyses. By using noninvasive tDCS it can further be analyzed whether the pain-related brain activity and connectivity changes can be normalized, demonstrating that these changes are not merely correlational, but causally related to the pain. This study therefore also attempts to unravel the fundamental underlying pathophysiology of fibromyalgia, an enigmatic constellation of symptoms.

\section{Methods}

\section{Participants}

Patients suffering from fibromyalgia were selected by the department of physical medicine and rehabilitation at the University Hospital Antwerp, Belgium, according to the criteria of the American College of Rheumatology (ACR)90 [27]. The inclusion and exclusion criteria for ACR-90 fibromyalgia diagnosis are as follows. The inclusion criteria were: 1) widespread body pain (present in the left, right, upper body, and lower body, as well as axial bone pain); and 2) tenderness at 11/18 defined trigger points [27]. The pain should not be explainable by other diseases (exclusion criteria) such as inflammatory arthritis (rheumatoid arthritis, polyarthritis and systemic disorder, systemic lupus erythematosus), axial skeletal syndromes (lower back pain syndromes and neck pain syndromes), osteoarthritis of knee or hand, nonarticular disorders (tendinitis and regional syndromes), or arthralgia syndromes [27]. The sensitivity of the ACR-90 criteria is $88.4 \%$ and the specificity $81.1 \%$ [27]. Thus, patients harboring pathologies mimicking the symptoms of fibromyalgia, as well as patients suffering from severe organic or psychiatric comorbidity (except minor depressive disorder), were excluded from participation. Furthermore, none of the patients were suffering from cervicotrigeminal tract radicular symptoms or types of hemicrania. 
Nineteen patients ( $4 \mathrm{men} / 15$ women) were included (mean \pm SD age 46.117 .85 years). All patients were intractable to tricyclic antidepressants (amitriptyline), pain medication, magnesium supplements, physical therapy, and psychological support. Most patients had tried one or more Food and Drug Administration-approved and off-label pain medications such as nonsteroidal anti-inflammatory drugs, opioids, duloxetine, flupentixol + melitracen, carbamazepine, gabapentine, and other antiepileptics, but not in a systematized way. Patients were allowed to stay on their current medications, and all patients agreed to make no changes to their current medication intake. All patients gave written informed consent and the ethical committee of the University Hospital Antwerp, Belgium, approved the study.

\section{Healthy Control Group}

Group age- and sex-matched EEG data of a healthy control group ( $n=19$; mean \pm SD age $46.2 \pm 7.64$ years) was collected. None of these subjects was known to suffer from tinnitus. Exclusion criteria were known psychiatric or neurologic illness, psychiatric history or drug/alcohol abuse, history of head injury (with loss of consciousness) or seizures, headache, or physical disability.

\section{tDCS}

Direct current (DC) was applied by a saline-soaked pair of surface sponges $\left(35 \mathrm{~cm}^{2}\right)$ and delivered by specially developed, battery-driven, constant current stimulator with a maximum output of $10 \mathrm{~mA}$ (Eldith ${ }^{\odot}$; see http://www.eldith.de). For each patient receiving tDCS 1 electrode was placed over the left (cathode) and one over the right (anode) $\mathrm{C} 2$ dermatome. A constant current of $1.5-\mathrm{mA}$ intensity was applied for 20 min. For sham tDCS, placement of the electrodes was identical to active tDCS. DC was first switched on in a ramp-up fashion over $5 \mathrm{~s}$. Current intensity (ramp down) was gradually reduced (over $5 \mathrm{~s}$ ) as soon as DC reached a current flow of $1.5 \mathrm{~mA}$. Hence, sham tDCS only lasted $10 \mathrm{~s}$ in comparison with $20 \mathrm{~min}$. The rationale behind this sham procedure was to mimic the transient skin sensation at the beginning of active tDCS, and it is assumed, yet unproven, that this does not produce any conditioning effects on the brain. The order of the sham and active tDCS was randomized over the different patients, but ultimately each patient underwent both the active tDCS and sham tDCS.

\section{Procedure}

After the pain percept evaluation tDCS treatment was initiated, and each patient underwent 1 consecutive week of sham tDCS treatment, and 1 week of active tDCS treatment with a 2-week washout between the sham and active treatment.
Patients were randomized for sham start/active treatment start and then subsequently crossed over to the other after the washout. For both the sham and the tDCS treatment, 1 week consisted of 3 sessions ( 1 session of 20 min every 2 days). After the final tDCS session and after the final tDCS sham session a pain perception evaluation was completed and provided to a blinded evaluator.

\section{Pain Evaluation}

\section{Primary Outcome Measure}

A numeric rating scale (NRS) for pain ("How much pain do you have? $0=$ no pain and $10=$ as painful as imaginable") was drawn before (pre-) and directly after (post-) active and sham tDCS stimulation. The NRS for pain has been compared in a functional analysis with other questionnaires that measure levels of pain, depression, overall mood, health, and so on., and has shown to have good correlations with all tests, indicating that the NRS for pain is a good tool to evaluate the overall impact of fibromyalgia [28].

\section{Secondary Outcome Measures}

The Fibromyalgia Impact Questionnaire measures the overall impact of fibromyalgia-related symptoms on the patient's life quality. The maximum score is 100; a higher score indicates a higher disease burden [29].

The Pain Catastrophizing Scale indicates the catastrophizing impact of pain experienced by the patient. It consists of 13 statements concerning pain experiences on a 5point scale [30].

\section{EEG Data}

\section{Collection and Preprocessing}

EEG data were obtained as a standard procedure. Recordings were obtained in a fully lit room, with each participant sitting upright on a small but comfortable chair. The actual recording lasted approximately $5 \mathrm{~min}$. The EEG was sampled using Mitsar-201 amplifiers (NovaTech, Mesa, AZ, USA) with 19 electrodes placed according to the standard 10-20 International placement (Fp1, Fp2, F7, F3, Fz, F4, F8, T7, $\mathrm{C} 3, \mathrm{Cz}, \mathrm{C} 4, \mathrm{~T} 8, \mathrm{P} 7, \mathrm{P} 3, \mathrm{Pz}, \mathrm{P} 4, \mathrm{P} 8, \mathrm{O} 1, \mathrm{O} 2$ ), analogous to what is was done in the normative group. Impedances were checked to remain below $5 \mathrm{k} \Omega$. Data were collected eyesclosed (sampling rate $=500 \mathrm{~Hz}$, band passed $0.15-200 \mathrm{~Hz}$ ). Off-line data were resampled to $128 \mathrm{~Hz}$, band-pass filtered in the range 2 to $44 \mathrm{~Hz}$, transposed into Eureka! software [31], plotted, and carefully inspected for manual artifact rejection. All episodic artifacts, including eye blinks, eye movements, teeth clenching, body movement, and ECG artifact were 
removed from the stream of the EEG. Average Fourier crossspectral matrices were computed for the traditionally used frequency bands: delta $(2-3.5 \mathrm{~Hz})$, theta $(4-7.5 \mathrm{~Hz})$, alpha1 $(8-10 \mathrm{~Hz})$, alpha2 $(10-12 \mathrm{~Hz})$, beta1 $(13-18 \mathrm{~Hz})$, beta2 $(18.5-21 \mathrm{~Hz})$, beta3 $(21.5-30 \mathrm{~Hz})$, and gamma $(30.5-44 \mathrm{~Hz})$.

\section{Source Localization}

Standardized low-resolution brain electromagnetic tomography (sLORETA [32]) was used to estimate the intracerebral electrical sources. As a standard procedure a common average reference transformation is performed before applying the sLORETA algorithm [32]. sLORETA computes electric neuronal activity as current density $\left(\mathrm{A} / \mathrm{m}^{2}\right)$ without assuming a predefined number of active sources. The solution space used in this study and associated lead-field matrix are those implemented in the LORETA-Key software (freely available at http://www.uzh.ch/keyinst/loreta.htm). This software implements revisited realistic electrode coordinates [33] and the lead field produced by [34] applying the boundary element method on the MNI-152 (Montreal Neurological Institute, Canada). The sLORETA-Key anatomic template divides and labels the neocortical (including hippocampus and anterior cingulated cortex) MNI-152 volume in 6239 voxels of $5 \mathrm{~mm}^{3}$ dimension, based on probabilities returned by the Demon Atlas [35]. The co-registration makes use of the correct translation from the MNI-152 space into the Talairach and Tournoux space.

\section{Region-of-Interest Pain Imbalance Measure}

The log-transformed electrical current density was averaged across all voxels belonging to the regions of interest. Regions of interest were the pregenual anterior cingulate cortex and the dorsal anterior cingulate cortex. To calculate the ratio, we divide the log-transformed current density from the pregenual anterior cingulate cortex by the dorsal anterior cingulate cortex for each frequency band separately.

\section{Lagged Phase Coherence}

Coherence and phase synchronization between time series corresponding to different spatial locations are usually interpreted as indicators of the "connectivity". However, any measure of dependence is highly contaminated with an instantaneous nonphysiological contribution due to volume conduction [36]. However, Pascual-Marqui [37] introduced new measures of coherence and phase synchronization taking into accounts only noninstantaneous (lagged) connectivity and therefore effectively removing the confounding factor of volume conduction. Such "lagged-phase coherence" between 2 sources can be interpreted as the amount of cross-talk between the regions contributing to the source activity [38]. As the two components oscillate coherently with a phase lag, the crosstalk can be interpreted as information sharing by axonal transmission. More precisely, the discrete Fourier transform decomposes the signal in a finite series of cosine and sine waves at the Fourier frequencies [39]. The lag of the cosine waves with respect to their sine counterparts is inversely proportional to their frequency and amounts to a quarter of the period; for example, the period of a sinusoidal wave at $10 \mathrm{~Hz}$ is $100 \mathrm{~ms}$. The sine is shifted a quarter of a cycle $(25 \mathrm{~ms})$ with the respect to the cosine. Then the lagged phase coherence at $10 \mathrm{~Hz}$ indicates coherent oscillations with a $25-\mathrm{ms}$ delay, while at $20 \mathrm{~Hz}$ the delay is $12.5 \mathrm{~ms}$, and so on. The threshold of significance for a given lagged phase coherence value according to asymptotic results can be found as described by Pascual-Marqui [36, 37], where the definition of lagged-phase coherence can also be found. As such, this measure of dependence can be applied to any number of brain areas jointly, that is, distributed cortical networks, whose activity can be estimated with sLORETA. The measures are non-negative, and take the value zero only when there is independence and are defined in the frequency domain: delta $(2-3.5 \mathrm{~Hz})$, theta $(4-7.5 \mathrm{~Hz})$, alpha1 $(8-10 \mathrm{~Hz})$, alpha2 $(10-12 \mathrm{~Hz})$, beta1 $(13-18 \mathrm{~Hz})$, beta2 $(18.5-21 \mathrm{~Hz})$, beta3 $(21.5-30 \mathrm{~Hz})$, and gamma $(30.5-44 \mathrm{~Hz})$. Based on this principle, lagged linear connectivity was calculated. Also, time series of current density were extracted for different region of interests using sLORETA. Power in all 6239 voxels was normalized to a power of 1 and log-transformed at each time point. Region of interest values thus reflect the logtransformed fraction of total power across all voxels, separately for specific frequencies. Regions of interest selected were the dorsal anterior cingulate cortex and the pregenual anterior cingulate cortex. The selection of these regions of interest was based on the model described in the "Introduction" (a priori) and confirmed by the comparison of activity between the different conditions for the fibromyalgia group and the comparison with control group (a posteriori).

\section{Granger Causality}

Granger causality measures the strength of effective connectivity, reflecting information transmission from one area to another by quantifying how much the signal in the seed region is able to predict the signal in the target region [40, 41]. In other words, it can be considered as a directional functional connectivity. Granger causality is defined as the log-ratio between the error variance of a reduced model, which predicts one-time series based only on its own past values, and that of the full model, which in addition includes the past values of another time series. It is important to note that Granger causality does not imply anatomical connectivity between regions but directional functional connectivity between two sources. 


\section{Statistical Analyses}

A repeated-measures analyis of variance (ANOVA) was conducted between baseline, sham, and active tDCS for the NRS for pain, Fibromyalgia Impact Questionnaire, and Pain Catastrophizing Scale. A correction for multiple comparisons was conducted between baseline, sham, and active tDCS using a Bonferroni correction. In addition, the ratio of the log-transformed current density between the pregenual anterior cingulate cortex and the dorsal anterior cingulate cortex was compared pre-versus active post-tDCS for each frequency band. To check if the ratio is normalized we compared the data with age- and sex-matched healthy controls without pain, using an independent $t$ test. In addition, the relationship between the subtraction of the pre-versus active post-tDCS log-transformed current density from the pregenual anterior cingulate cortex by the dorsal anterior cingulate cortex and the subtraction between pain scores (NRS for pain, Fibromyalgia Impact Questionnaire, and Pain Catastrophizing Scale) of the pre- versus active posttDCS, and pre- versus sham post-tDCS was computed using a Pearson correlation for each frequency band separately. To further compare the active and sham tDCS correlations we computed the Hotelling's $t$ test for correlated correlations within a population and the Steiger's $Z$ test for correlated correlations within a population. The second technique has the advantage that it corrects for the overestimated $t$ value (resulting in a type I error) by using Fisher's transformation, changing $r$ to a $Z$ score. The technique used to compare active and sham tDCS correlations was also applied for the connectivity meausres.

The methodology used for EEG data analysis is a nonparametric permutation test. It is based on estimating, via randomization, the empirical probability distribution for the max-statistic under the null hypothesis comparisons [42]. This methodology corrects for multiple testing (i.e., for the collection of tests performed for all voxels, and for all frequency bands). Owing to the nonparametric nature of this method, its validity does not rely on any assumption of Gaussianity [42]. The significance threshold for all tests was based on a permutation test with 5000 permutations. Comparisons were made between the baseline, sham, and active tDCS stimulation. These comparisons were performed on a whole brain by sLORETA statistical contrast maps through multiple voxel-by-voxel comparisons in a logarithm of $F$-ratio.

The log-transformed electrical current density was averaged across all voxels belonging to the regions of interest. Regions of interest were the pregenual anterior cingulate cortex and the dorsal anterior cingulate cortex. To calculate the ratio, we divide the log-transformed current density from the pregenual anterior cingulate cortex by the dorsal anterior cingulate cortex for each frequency band separately.
A comparison was made between baseline, sham, and active tDCS for the patients with fibromyalgia on Granger causality outcome measures using a repeated-measure ANOVA. Pearson correlations were calculated between the Granger causality outcome measures per patient and, respectively, the NRS, Fibromyalgia Impact Questionnaire, and the Pain Catastrophizing Scale on lagged phase coherence outcome.

\section{Results}

\section{Neurophysiological Changes in Fibromyalgia (Baseline) in Comparison with Healthy Subjects}

\section{Activity}

A comparison between patients with fibromyalgia and healthy controls revealed a significant effect $(p<0.05)$ for the beta 2 and beta 3 frequency bands. For both frequency bands an increased activity was identified for patients with fibromyalgia in the dorsal anterior cingulate cortex in comparison with healthy subjects (see Fig. 1, top panel). No significant effects were elicited for the delta, theta, alpha1, alpha2, beta1, and gamma frequency bands.

A correlation analysis between brain activity and, respectively, the NRS, Fibromyalgia Impact Questionnaire, and the Pain Catastrophizing Scale revealed no significant effects for the delta, theta, alpha1, alpha2, beta1, beta2, beta3, and gamma frequency bands. In addition, a region-of-interest correlation analysis looking at the association between the dorsal anterior cingulate and the NRS, the Fibromyalgia Impact Questionnaire, and the Pain Catastrophizing Scale, respectively, showed no significant effect.

A comparison between the balance of the log-transformed current density between the pregenual anterior cingulate cortex and the dorsal anterior cingulate cortex revealed a significant effect for the alpha1 $(t=2.41, p=0.023)$, beta2 $(t=2.11$, $p=0.031)$, and beta3 $(t=2.09, p=0.047)$ frequency bands (see Fig. 1, mid-panel). It was demonstrated that patients with fibromyalgia are characterized by a lower score on the pain balance (pgACC/dACC current density) in comparison with healthy controls for alpha1, beta2, and beta3. This suggests that for alpha1, beta2, and beta3 the current density was low in the pregenual anterior cingulate cortex and high in the dorsal anterior cingulate cortex for the patients with fibromyalgia at baseline in comparison with the healthy control subjects. No significant effects were obtained for the delta, theta, alpha2, beta1, and gamma frequency bands.

\section{Lagged-Phase Coherence}

A lagged-phase synchronization analysis revealed a significantly $(p<0.05)$ decreased functional connectivity 
Activity analysis: Fibromyalgia (baseline) vs healthy controls

beta2

$\mathbf{L}$
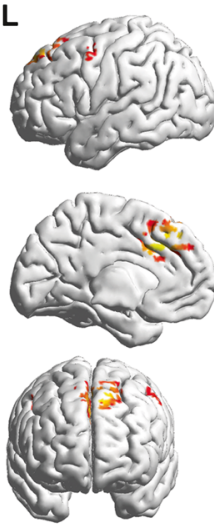
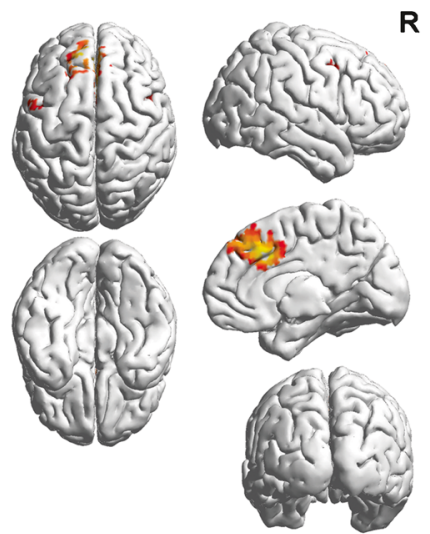

beta3
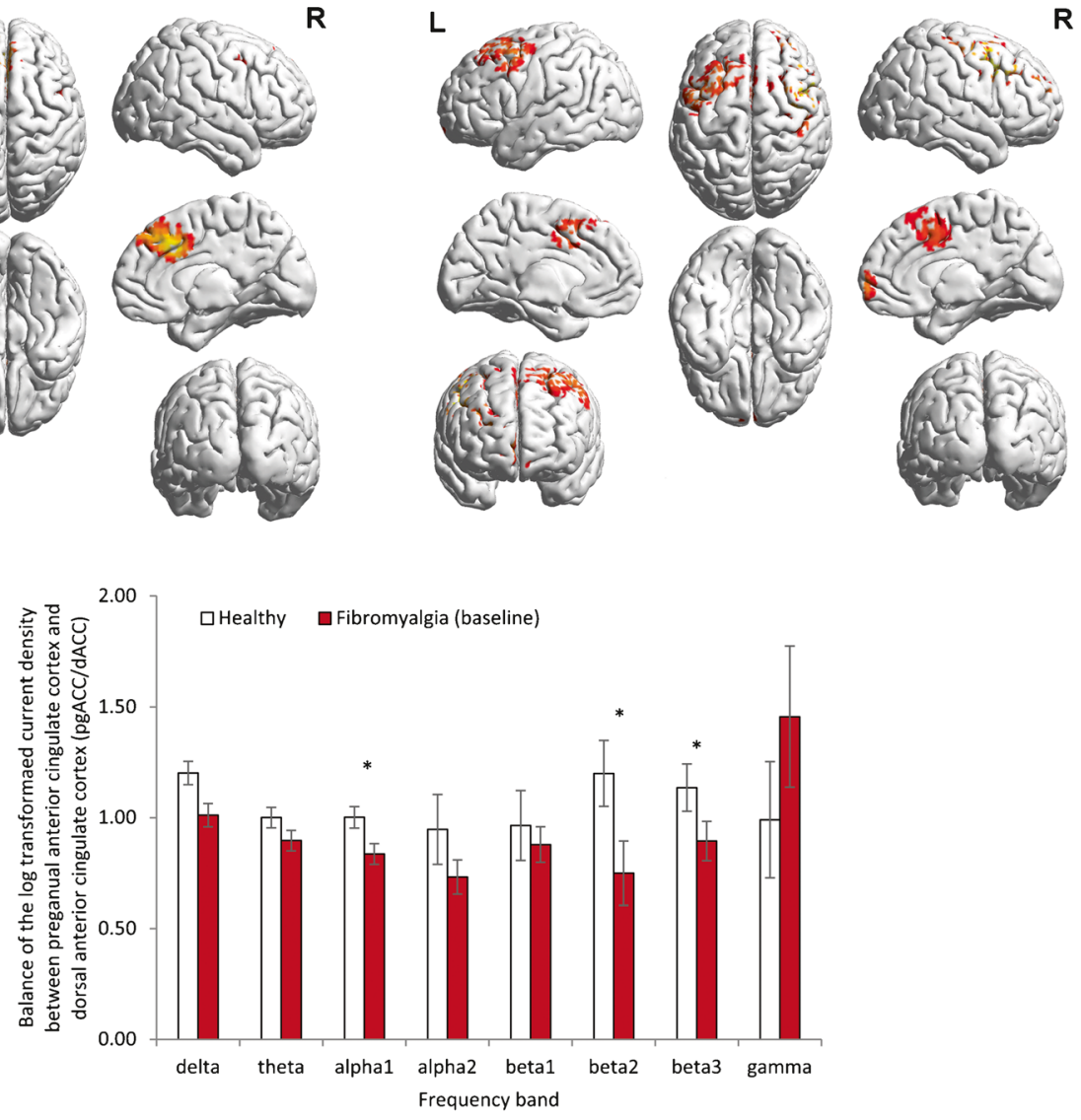

Connectivity analysis: Fibromyalgia (baseline) vs healthy controls
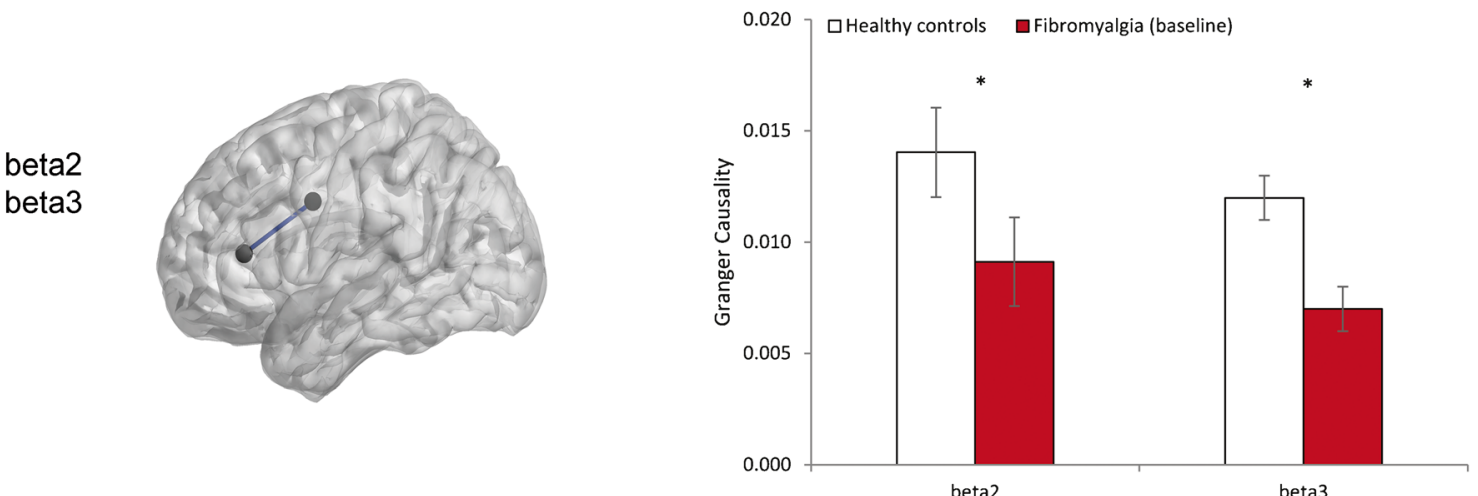

Fig. 1 A comparison between patients with fibromyalgia at baseline and healthy control subjects shows a significant difference in activity (top panel), in the balance between the pregenual anterior

cingulate cortex and dorsal anterior cingulate cortex (mid-panel), in connectivity and Granger causality (lower panel)

between the dorsal anterior cingulate cortex and the pregenual anterior cingulate cortex for patients with fibromyalgia in comparison with healthy controls in the beta2 and beta3 frequency bands (see Fig. 1, mid-panel). No effect was obtained for the delta, theta, alpha1, alpha2, beta1, and gamma frequency bands.

A significant negative correlation was found for the functional connectivity strength between the dorsal anterior cingulate cortex and the pregenual anterior cingulate cortex with NRS $(r=-0.43, p<0.01)$ but not for the Fibromyalgia Impact Questionnaire $(r=-0.37, p<0.51)$ or the Pain Catastrophizing Scale $(r=-0.32, p<0.51)$ for the beta2 
frequency band (see Fig. 4). No effect was obtained for the delta, theta, alpha1, alpha2, beta1, beta3, and gamma frequency bands.

\section{Granger Causality}

A repeated-measure ANOVA revealed a significant main effect $(F=8.70, p=0.006)$ for group (healthy $v s$ baseline fibromyalgia), indicating that patients with fibromyalgia have a lower score than healthy controls (see Fig. 1, lower panel). For the beta 2 frequency band no significant effect was identified for the main effect, direction of connectivity (dorsal anterior cingulate cortex to pregenual anterior cingulate cortex $v S$ pregenual anterior cingulate cortex to dorsal anterior cingulate cortex), and for the interaction effect group $\times$ direction of connectivity for the beta2 frequency band.

A similar analysis for the beta3 frequency band revealed a significant main effect $(F=6.57, p=0.015)$ for group (healthy $v s$ baseline fibromyalgia), indicating that patients with fibromyalgia have a lower score than healthy controls for the beta 3 frequency band (see Fig. 1, lower panel). For the beta3 frequency band no significant effect was obtained for the main effect, direction of connectivity, and for the interaction effect group $\times$ direction of connectivity.

\section{Behavioral Changes Obtained by tDCS in Fibromyalgia}

A repeated-measures ANOVA revealed a significant effect between the 3 conditions (baseline, sham, and active) for the NRS for pain $(F=23.14, p<0.001)$. A pairwise comparison between baseline and, respectively, the sham tDCS $(p<0.001)$ or active tDCS $(p<0.001)$ showed, in both cases, a significant effect after Bonferroni correction. This showed a suppression effect of $11.66 \%$ after sham tDCS and $28.09 \%$ after active tDCS. A comparison between sham tDCS and active tDCS also revealed a significant effect ( $p=0.007)$, indicating a suppression effect of $18.60 \%$ for the active tDCS in comparison with sham tDCS (see Fig. 2 for an overview).

For the Fibromyalgia Impact Questionnaire, a significant effect was identified between the 3 conditions (baseline, sham, and active) $(F=33.24, p<0.001)$. A pairwise comparison after Bonferroni correction showed that active tDCS was significantly different than the baseline $(p<0.001)$ and sham tDCS $(p<0.001)$. A comparison between baseline and sham also revealed a significant effect $(p<0.001)$. In comparison with baseline a suppression effect of $9.79 \%$ was obtained for active tDCS, while in comparison with sham stimulation $7.01 \%$ (see Fig. 2 for an overview).

A comparison between the 3 conditions (baseline, sham, and active) for the Pain Catastrophizing Scale revealed a significant effect $(F=19.17, p<0.001)$. A pairwise comparison between baseline and, respectively, the sham tDCS $(p=0.003)$. and active tDCS $(p<0.001)$ showed in both cases a significant effect after Bonferroni correction $(p<0.001)$. This showed a suppression effect of $2.92 \%$ after sham tDCS and $9.69 \%$ after active tDCS. A comparison between sham tDCS and active tDCS also revealed significant effect ( $p=$ 0.001 ), indicating a suppression effect of $6.98 \%$ for the active tDCS in comparison with sham tDCS (see Fig. 2 for overview).

\section{Neurophysiological Changes Obtained by tDCS}

\section{Activity}

A comparison between baseline and active tDCS in patients with fibromyalgia yielded a significant effect $(p<0.05)$ for the beta1, beta2, beta3, and gamma frequency bands (see Fig. 3). For the beta1, beta2, beta3, and gamma frequency bands, increased activity was noted in the pregenual anterior cingulate cortex/ventral medial prefrontal cortex extending to the left ventrolateral and dorsolateral prefrontal cortex after active tDCS in comparison with baseline. In addition, significantly reduced activity over the dorsal anterior cingulate cortex extending to the sensorimotor area was found after active tDCS in comparison with baseline for beta 2 and beta 3 frequency bands. No significant effects were elicited for the delta, theta, alpha1, and alpha2 frequency bands.

A comparison between baseline and sham tDCS in patients with fibromyalgia revealed no significant effect for the delta, theta, alpha1 alpha2, beta1, beta2, beta3, and gamma frequency bands.

For the beta1, beta2, and gamma frequency bands a significant effect $(p<0.05)$ was obtained when comparing sham tDCS and active tDCS in patients with fibromyalgia (see Fig. S1). For the beta1 and beta2 frequency bands, increased activity in the pregenual anterior cingulate cortex/ventral medial prefrontal cortex extending to the left ventrolateral prefrontal cortex and left insula was elucidated after active tDCS in comparison with sham tDCS. For the gamma frequency band, increased activity was found in the left motor cortex. No significant effect was seen for the delta, theta, alpha1, alpha2, and beta3 frequency bands. A comparison between sham tDCS for patients with fibromyalgia versus healthy control subjects showed a significant effect for the beta3 frequency band at the dorsal anterior cingulate cortex for the patients with fibromyalgia after sham tDCS (see Fig. S1). No significant effect was seen for the delta, theta, alpha1 alpha2, beta1, beta2, and gamma frequency bands.

A comparison between the balance of the log-transformed current density between the pregenual anterior cingulate cortex and the dorsal anterior cingulate cortex revealed a significant effect for the delta $(t=2.09, p=0.03)$, theta $(t=1.81$, $p=0.04)$, and beta3 $(t=1.92, p=0.04)$ frequency bands (see Fig. 4, top panel). It was demonstrated that after active tDCS a higher score in balance was obtained in comparison with 

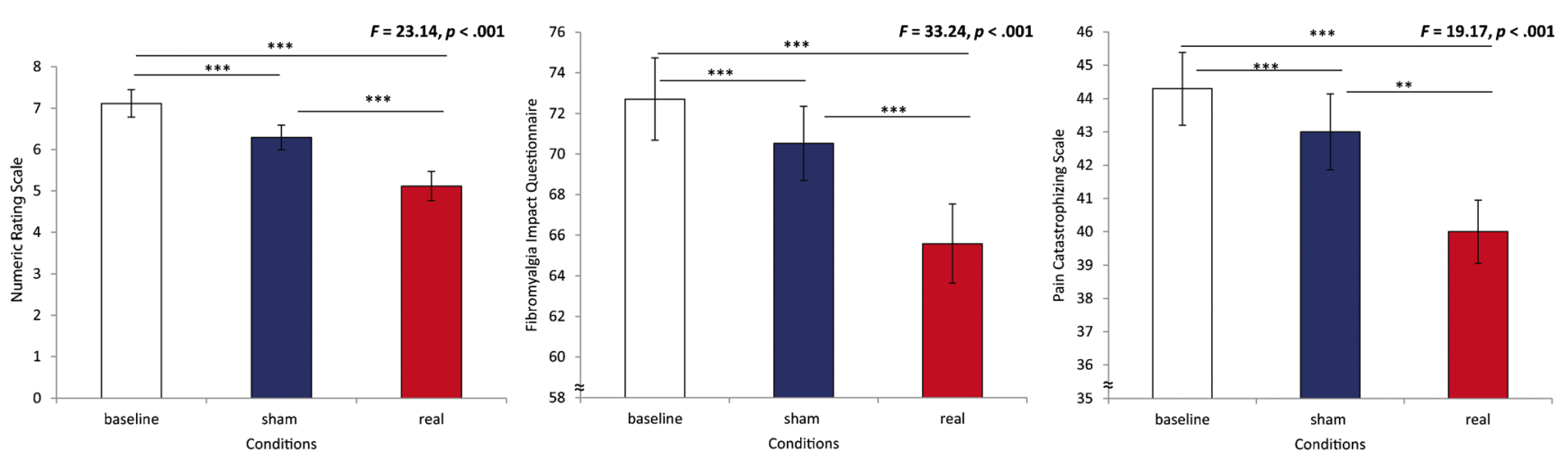

Fig. 2 A comparison between baseline, sham transcranial direct current stimulation (tDCS) and active tDCS shows a significant pain reduction for active tDCS in comparison with sham tDCS and baseline for the numeric rating scale, Fibromyalgia Impact Questionnaire,

and Pain Catastrophizing Scale. Sham tDCS is also significantly better than baseline for the numeric rating scale (left), Fibromyalgia Impact Questionnaire (middle), and Pain Catastrophizing Scale (right). $* \mathrm{p}<.05, * * \mathrm{p}<.01, * * * \mathrm{p}<.001$

baseline, signifying a shift towards more pain inhibition in relation to pain sensitization. No significant effects were obtained for the alpha1, alpha2, beta1, beta2, and gamma frequency bands.

Correlation analysis between baseline and active tDCS for the balance of the pregenual anterior cingulate cortex and dorsal anterior cingulate cortex (= pregenual anterior cingulate cortex/dorsal anterior cingulate cortex), and difference between baseline and active tDCS pain score on the NRS demonstrated a positive correlation for the gamma frequency band $(\mathrm{r}=0.66, p=0.003)$. This correlation showed that the higher the difference between the pre- and postbalance, the higher the pain reduction was, or vice versa. A similar effect was obtained for the Fibromyalgia Impact Questionnaire $(\mathrm{r}=0.49, p=0.03)$ and the Pain Catastrophizing Scale $(r=.76, p<0.001)$ for the gamma frequency band. For both the NRS and fibromyalgia impact Questionnaire, no significant effects were obtained for the delta, theta, alpha1, alpha2, beta1, beta2, and beta3 frequency bands. However, for the Pain Catastrophizing Scale $(r=0.49, p<0.03)$ an effect was also shown for the beta3 frequency band. If we controlled for the amount of frequency bands using a Bonferroni correction, only the effect for NRS for pain and the Pain Catastrophizing Scale regarding the gamma frequency remained. No significant effects were obtained for difference between the pre- and sham tDCS condition, and the balance of the pregenual anterior cingulate cortex and dorsal anterior cingulate cortex (= pregenual anterior cingulate cortex/ dorsal anterior cingulate cortex) revealed no significant effects for the delta, theta, alpha1, alpha2, beta1, beta2, beta3, and gamma frequency bands. In addition, a comparison between the active and sham correlations for the gamma

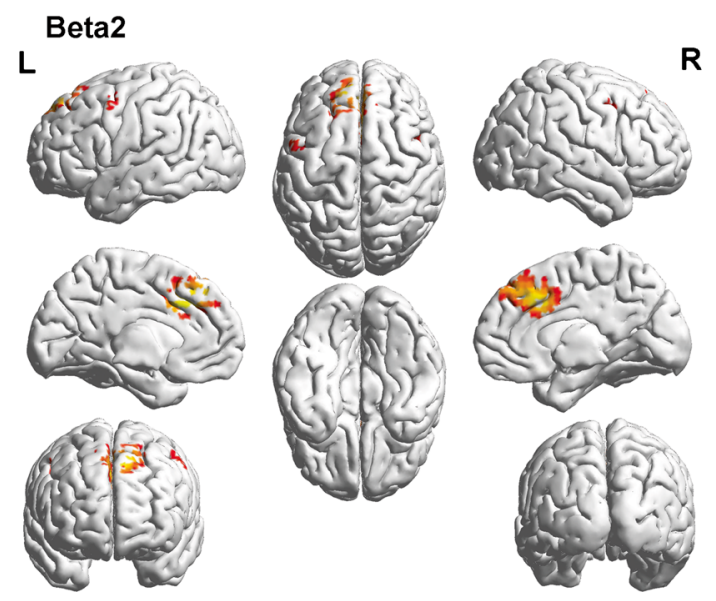

Fig. 3 A comparison between baseline and active transcranial direct current stimulation (tDCS) demonstrates that active tDCS is associated with increased activity in the beta 1 , beta2, beta3, and gamma frequency bands in the pregenual anterior cingulate cortex/ventral medial

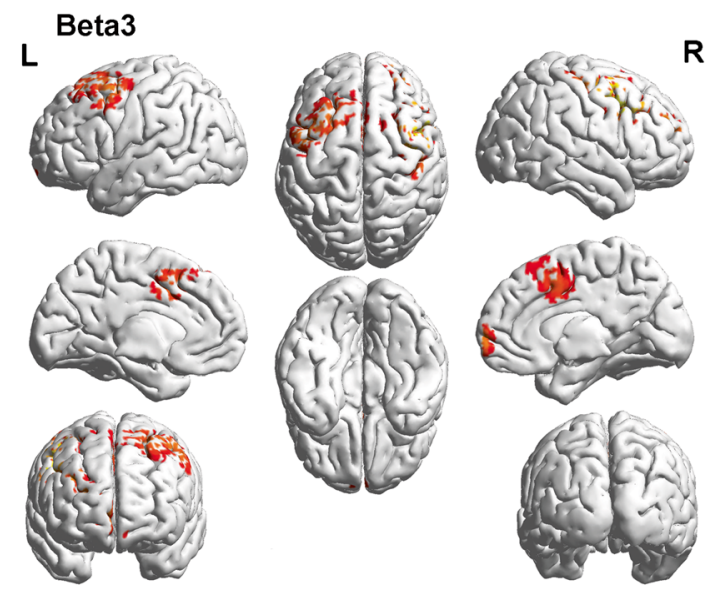

prefrontal cortex extending to the ventral lateral prefrontal cortex/ dorsal lateral prefrontal cortex. Active tDCS is also associated with decreased beta activity in the dorsal anterior cingulate cortex/ supplementary motor cortex extending to the sensorimotor cortex 

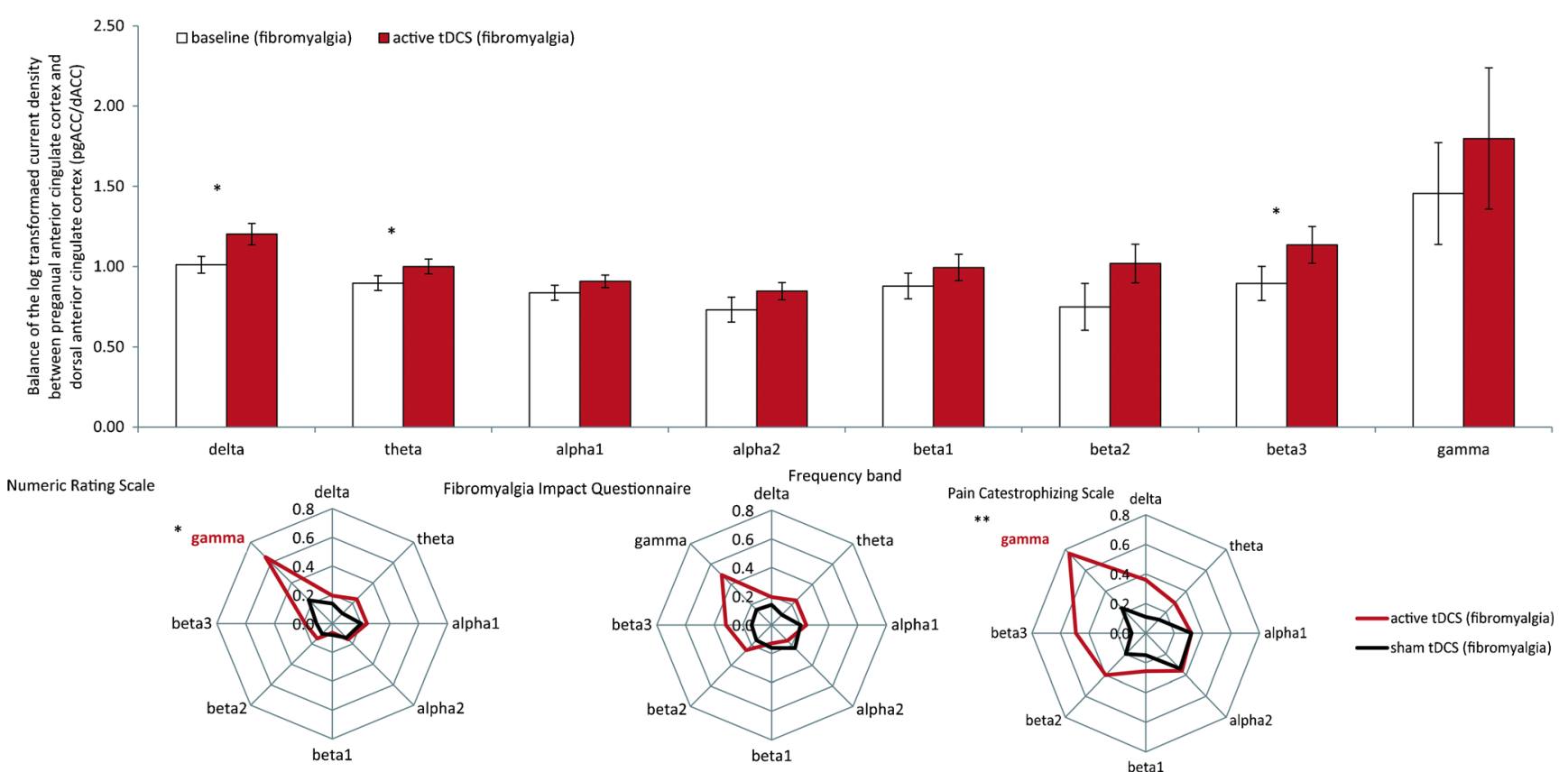

Fig. 4 Comparison between baseline and active transcranial direct current stimulation (tDCS) for the balance of the pregenual anterior cingulate cortex and dorsal anterior cingulate cortex (top panel). A comparison between patients with fibromyalgia (post-active tDCS) and health control subjects (below). Correlation between baseline and post-tDCS for the balance of the pregenual anterior cingulate cortex and dorsal anterior cingulate cortex and difference between the pre-, sham tDCS, and active tDCS for visual analog score for pain, Fibromyalgia Impact Questionnaire, and Pain Catastrophizing Scale frequency band revealed a significant difference for the NRS for pain $(t=1.88, p=0.04 ; Z=1.69, p=0.045)$ and the Pain Catastrophizing Scale $(t=2.64, p=0.009 ; Z=2.25, p=0.01)$ but not for the Fibromyalgia Impact Questionnaire $[t=1.31$, $p=0.10 ; Z=1.24, p=0.10$ (see Fig. 4, lower panel)].

\section{Lagged-Phase Coherence}

A coherence analysis revealed a significant difference $(p<0.05)$ between baseline and active tDCS for the beta2, beta3, and gamma frequency bands, thus indicating an increased connectivity between the pregenual anterior cingulate cortex and dorsal anterior cingulate cortex after active tDCS (see Fig. 5, top panel). No significant effects were seen for the delta, theta, alpha1, alpha2, and beta1 frequency bands.

A comparison between sham tDCS in fibromyalgia and baseline yielded no significant effect for the connectivity for the delta, theta, alpha1 alpha2, beta1, beta2, beta3, and gamma frequency bands.

Between active tDCS and sham tDCS in fibromyalgia, a comparison showed a significant effect $(p<0.05)$ for the beta2, beta3, and gamma frequency bands, revealing an increased functional connection between the pregenual anterior cingulate cortex and dorsal anterior cingulate cortex after active tDCS. No significant effects were obtained for the delta, theta, alpha1, alpha2, and beta1 frequency bands.
A correlation analysis between the connectivity strength between the pregenual anterior cingulate cortex and dorsal anterior cingulate cortex and, respectively, the NRS, Fibromyalgia Impact Questionnaire, and the Pain Catastrophizing Scale showed a significant effect for the beta1, beta2, and beta3 frequency bands for active tDCS (see Fig. 5, mid panel). No significant effects were obtained for the delta, theta, alpha1, alpha2, and gamma frequency bands.

A correlation analysis between the connectivity strength between the pregenual anterior cingulate cortex and dorsal anterior cingulate cortex, and, respectively, the NRS, Fibromyalgia Impact Questionnaire, and the Pain Catastrophizing Scale demonstrated no significant effect for the sham tDCS for the delta, theta, alpha1, alpha2, beta1, beta2, beta3, and gamma frequency bands.

A comparison between the active and sham correlations revealed a significant difference for the NRS for pain for beta 1 , beta 2 , and beta 3 frequency bands. For the Pain Catastrophizing Scale no significance was found, but not for the Fibromyalgia Impact Questionnaire an effect was obtained for the beta 2 frequency band.

A correlation analysis between changes in connectivity (active tDCS - baseline) and, respectively, the amount of reduction on the NRS, Fibromyalgia Impact Questionnaire, and the Pain Catastrophizing Scale (baseline-active tDCS) revealed a positive correlation for the beta 2 frequency band. 
Connectivity analysis

Active $\mathrm{tDCS}$ vs Baseline (fibromyaglia)

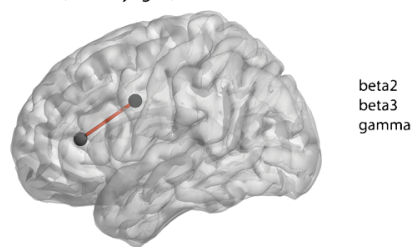

Correlation analyis belavioratmeasueand conectivity strenght fibonyalia) Numeric Rating Scale
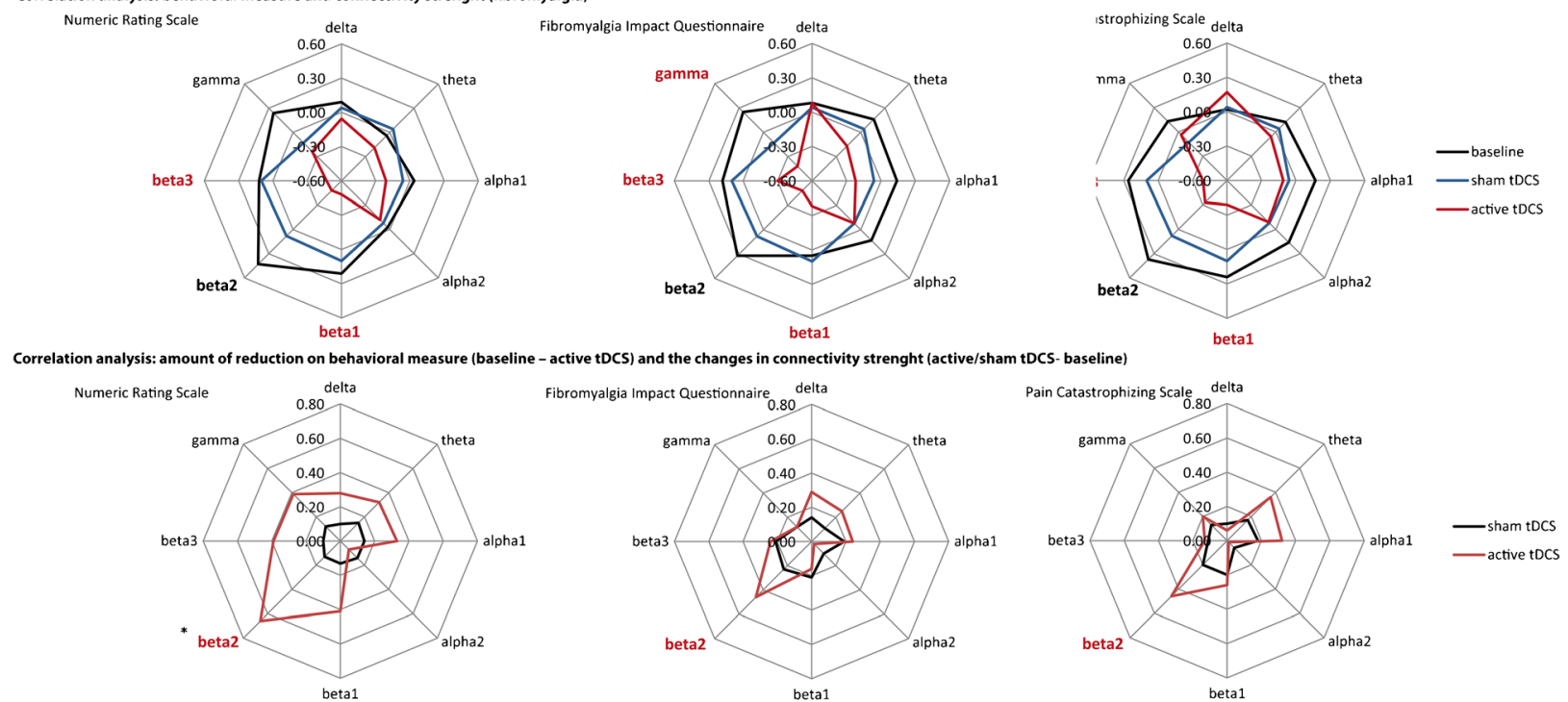

Granger Causality for the beta2 frequency band
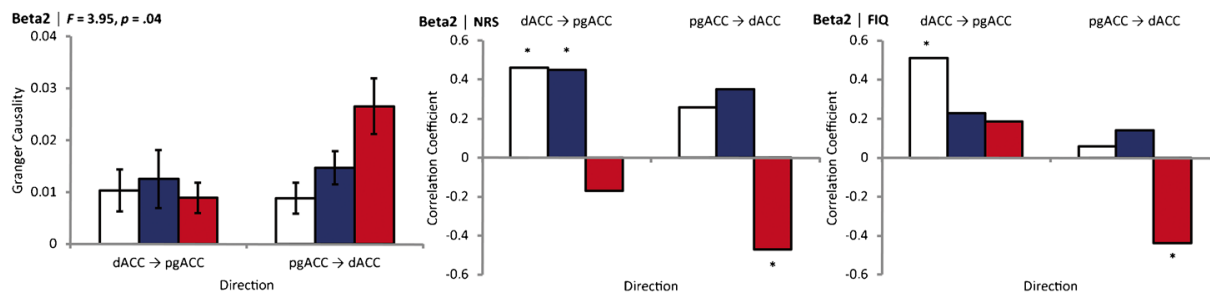

eta2 | PCS dACC $\rightarrow$ pgACC

$\mathrm{PgACC} \rightarrow \mathrm{dACC}$
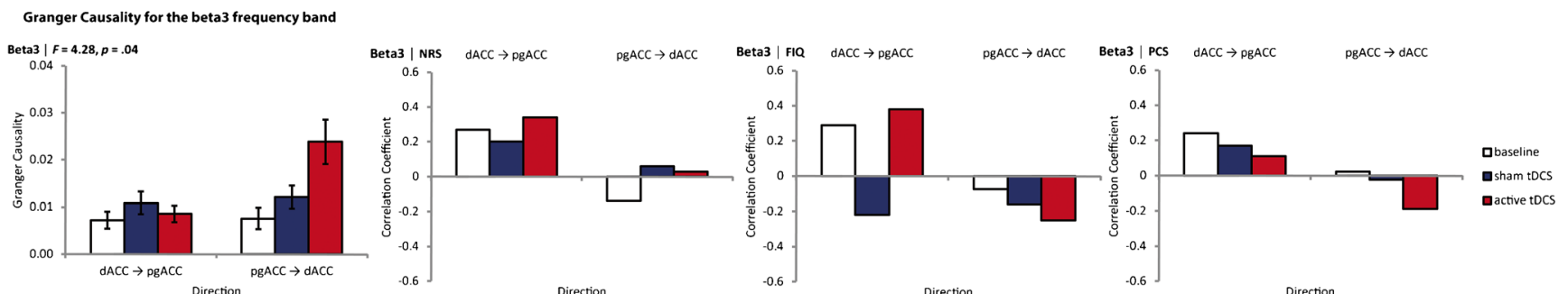

Fig. 5 A comparison between baseline, sham, and active transcranial direct current stimulation (tDCS) for the connectivity (top panel), the correlation between beharvioral measures (visual analog score for pain, Fibromyalgia Impact Questionnaire, and Pain Catastrophizing Scale) and connectivity strength, the correlation on the amount of reduction for visual analogue score for pain, Fibromyalgia Impact Questionnaire and

Pain Catastrophizing Scale, and connectivity strength. Granger causalitity for the beta2 and beta3 frequency band between the pregenual anterior cingulate cortex and dorsal anterior cingulate cortex, as well as the correlation between Granger causlity and visual analog score for pain [numeric rating scale (NRS)], Fibromyalgia Impact Questionnaire (FIQ), and Pain Catastrophizing Scale (PCS)

No significant effects were obtained for the delta, theta, alpha1, alpha2, beta 1 , beta3, and gamma frequency bands (see Fig. 5). In addition, a correlation analysis for the amount of reduction between the changes in connectivity (baselinesham tDCS) and, respectively, the NRS, Fibromyalgia
Impact Questionnaire, and the Pain Catastrophizing Scale (baseline-sham tDCS) identified no significant effects.

A comparison between the active and sham correlations revealed a significant difference for the NRS for pain for the beta 2 and beta 3 frequencybands. ForthePain Catastrophizing 
Scale and Fibromyalgia Impact Questionnaire no significant effects were obtained.

\section{Granger Causality}

A repeated-measures ANOVA revealed a significant interaction effect $(F=3.95, p=0.044)$ between condition (baseline, sham, or active tDCS) and direction of connectivity (dorsal anterior cingulate cortex to pregenual anterior cingulate cortex $v s$ pregenual anterior cingulate cortex to dorsal anterior cingulate cortex) for the beta2 frequency band (see Fig. 5, lower panel). Our results show that for both baseline and sham tDCS significantly more information was sent from the dorsal anterior cingulate cortex to pregenual anterior cingulate cortex in comparison with active tDCS. However, for the pregenual anterior cingulate cortex to dorsal anterior cingulate cortex significantly more information was sent after active tDCS comparison with both baseline and sham tDCS.

For the beta2 frequency band, a Pearson correlation was computed between the direction of connectivity (dorsal anterior cingulate cortex to pregenual anterior cingulate cortex or pregenual anterior cingulate cortex to dorsal anterior cingulate cortex) and the 3 measures of pain. For the NRS, a significant correlation was found for the baseline $(r=0.46, p=0.035)$ and sham tDCS $(r=0.45, p=0.036)$ condition but not for active tDCS. This indicates that the more information was sent from the dorsal anterior cingulate cortex to pregenual anterior cingulate cortex, the more pain the patients with fibromyalgia perceived. For the Fibromyalgia Impact Questionnaire, a significant correlation was identified for baseline $(r=0.51, p=0.021)$ and for the direction dorsal anterior cingulate cortex to pregenual anterior cingulate cortex but not for the sham and active tDCS. For the pregenual anterior cingulate cortex to dorsal anterior cingulate cortex direction, a significant effect was obtained with the NRS $(r=-0.47, p=0.034)$ and the Fibromyalgia Impact Questionnaire $(r=-0.44, p=0.045)$ for active tDCS, indicating the more information was sent from the pregenual anterior cingulate cortex to dorsal anterior cingulate cortex the less pain the patients with fibromyalgia perceived. No effects were obtained for the Pain Catastrophizing Scale.

A similar effect was obtained for the beta3 frequency band demonstrating a significant interaction effect $(F=$ $4.26, p=0.036$ ) between condition (baseline, sham, or active tDCS) and direction of connectivity (dorsal anterior cingulate cortex to pregenual anterior cingulate cortex vs pregenual anterior cingulate cortex to dorsal anterior cingulate cortex). Analogous to the beta 2 frequency band, the results revealed that for both baseline and sham tDCS, significantly more information was sent from the dorsal anterior cingulate cortex to pregenual anterior cingulate cortex in comparison with active tDCS. For the pregenual anterior cingulate cortex to dorsal anterior cingulate cortex, significantly more information was sent after active tDCS in comparison with both baseline and sham tDCS. No effect was obtained for both main effects. A Pearson correlation between the direction of connectivity (dorsal anterior cingulate cortex to pregenual anterior cingulate cortex or pregenual anterior cingulate cortex to dorsal anterior cingulate cortex) and the 3 measure of pain evaluation (NRS, Fibromyalgia Impact Questionnaire, and Pain Catastrophizing Scale) revealed no significant effects for the beta3 frequency band (see Fig. 5, lower panel).

\section{Normalization of Activity and Connectivity}

A comparison between patients with fibromyalgia after active tDCS and healthy controls revealed a significant effect $(p<0.05)$ for the beta 2 and beta 3 frequency bands (see Fig. 6). For both frequency bands increased activity was found for patients with fibromyalgia in the pregenual anterior cingulate cortex in comparison with healthy subjects. No significant effects were elicited for the delta, theta, alpha1, alpha2, beta1, and gamma frequency bands.

A comparison between patients with fibromyalgia and healthy control subjects for the log-transformed current density between the pregenual anterior cingulate cortex and the dorsal anterior cingulate cortex showed no significant effect for the delta, theta, and beta3 frequency bands after tDCS (see Fig. 6, mid-panel).

A lagged-phase synchronization analysis revealed a significantly $(p<0.05)$ increased functional connectivity between the dorsal anterior cingulate cortex and the pregenual anterior cingulate cortex for patients with fibromyalgia after active tDCS in comparison with healthy controls in the beta2, beta3, and gamma frequency bands (see Fig. 6, mid-panel). No effect was obtained for the delta, theta, alpha1, alpha2, and beta1 frequency bands.

A comparison for effective connectivity revealed a significant interaction effect between condition (baseline, sham, or active tDCS) and direction of connectivity (dorsal anterior cingulate cortex to pregenual anterior cingulate cortex $v s$ pregenual anterior cingulate cortex to dorsal anterior cingulate cortex $)$ for the beta2 $(F=6.88, p=0.013)$ and beta3 $(F=7.79$, $p=0.009$ ) frequency bands. A main effect was obtained for the beta2 $(F=10.57, p=0.013)$ and beta3 $(F=11.23, p=$ 0.002 ) frequency band, revealing that patients with fibromyalgia have larger Granger causality than healthy controls (see Fig. 6, lower panel).

For the direction dorsal anterior cingulate cortex to pregenual anterior cingulate cortex, no significant changes were identified between healthy controls and patients with fibromyalgia. For the direction pregenual anterior cingulate cortex to dorsal anterior cingulate cortex, patients with 
Fibromyalgia activity analysis: active tDCS vs healthy controls

Beta2
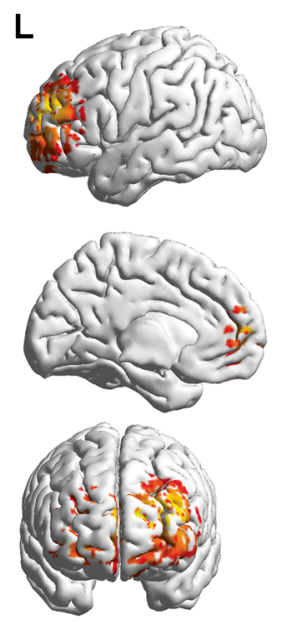

\section{Beta3}

R L
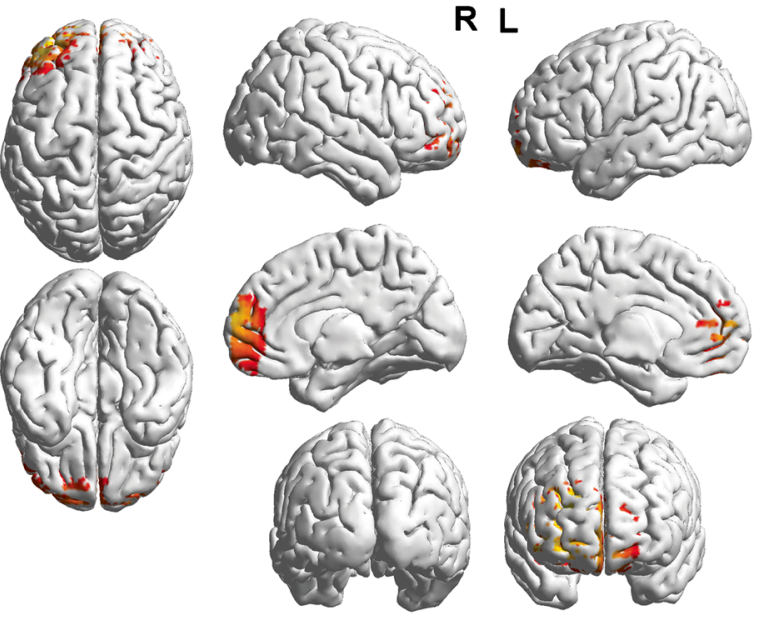
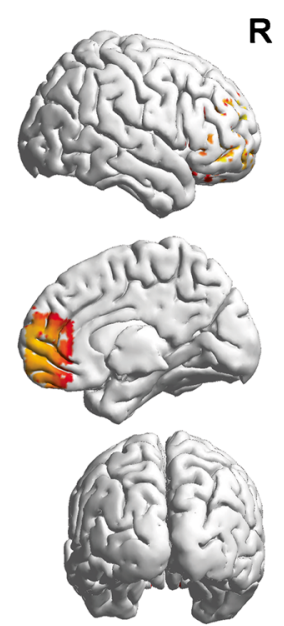

$\mathbf{R}$

\section{Lagged phase coherence}

Active tDCS (fibromyalgia) vs Healthy Controls
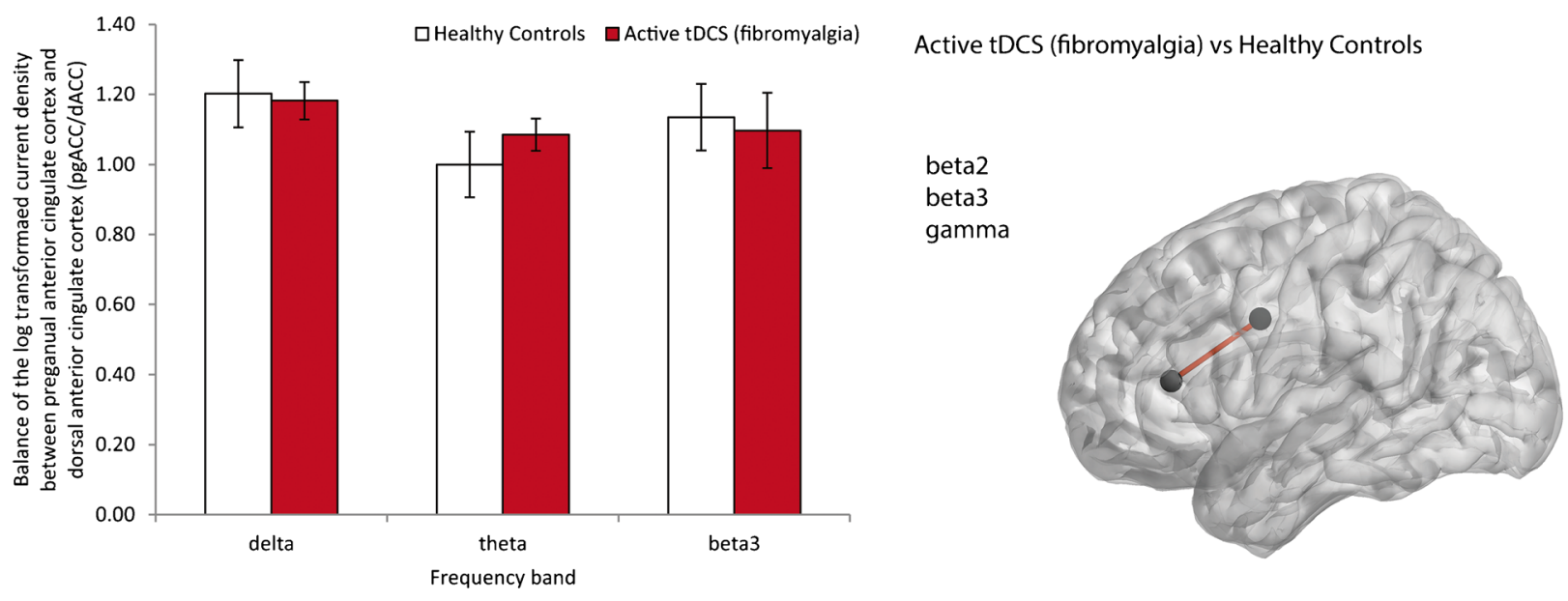

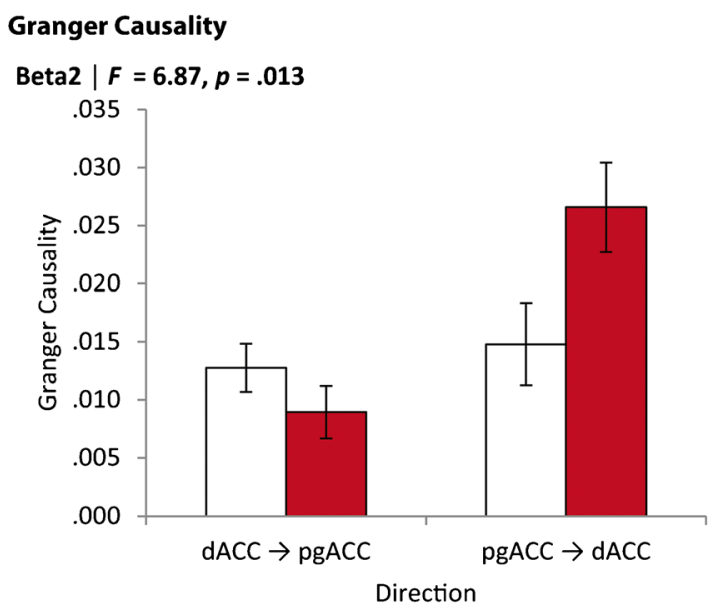

Fig. 6 Comparison between active transcranial direct current stimulation (tDCS) for patients with fibromyalgia and healthy control subjects for activity (top panel), the balance between the

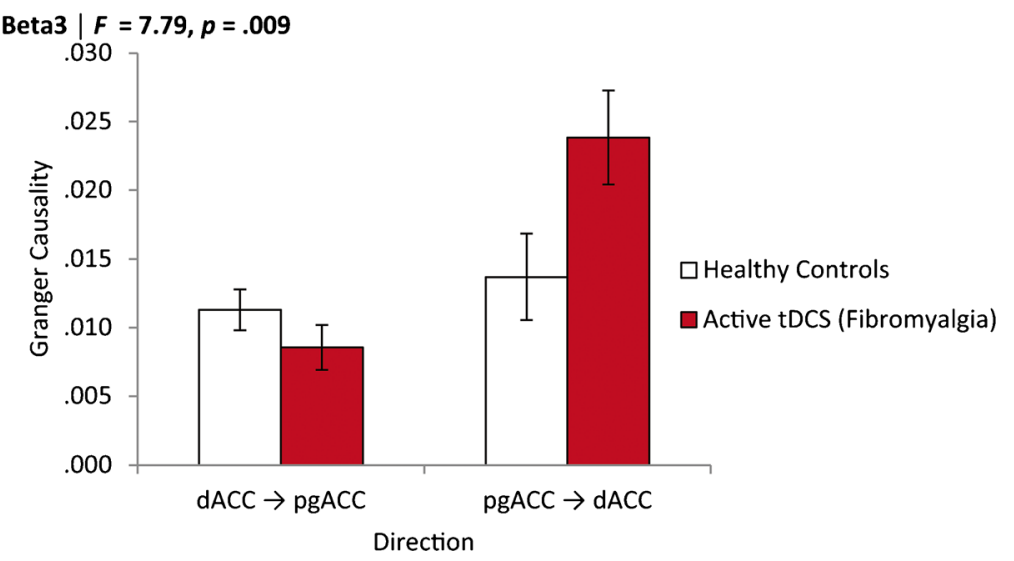

pregenual anterior cingulate cortex and dorsal anterior cingulate cortex (mid-panel), connectivity (mid panel), and Granger causality (lower panel) 
fibromyalgia showed stronger Granger causality in comparison with healthy subjects. A main effect was obtained for the beta2 $(F=9.72, p=0.004)$ and beta3 $(F=12.10, p=0.001)$ frequency bands showing an effect for direction of connectivity (see Fig. 6, lower panel). That is, Granger causality is stronger from pregenual anterior cingulate cortex to dorsal anterior cingulate cortex in comparison with dorsal anterior cingulate cortex to pregenual anterior cingulate cortex.

\section{Safety and Complications}

tDCS was well tolerated and no tDCS-related complications, except for transient redness, and slight itching, were noted by the patients during or after the tDCS sessions. More specifically, no skin lesions, the most common complication of tDCS, were noted [43].

\section{Discussion}

\section{Central Mechanisms in Pain Perception}

Pain is encoded by at least 3 pathways, 2 ascending paindetecting pathways $[44,45]$ and at least 1 descending paininhibitory pathway [6]. The medial pain pathway encodes the motivational/affective component of pain $[44,45]$ clinically expressed as unpleasantness [45, 46]. The lateral pathway discriminates the sensory [45] aspects of pain, that is, the localization, intensity, and character (e.g., burning, sharp, dull) of the pain. The descending pathway decreases ongoing pain in a state-dependent manner [6]. The medial and lateral pain pathways are processed in parallel [47], and can be individually modified without affecting the other pathway [45], even though commonly both go hand in hand. The motivational ascending medial pain pathway and the state-dependent descending paininhibitory pathway are both contextually influenced [48]. The ascending medial system is activated by C-fibers and connects to the mediodorsal and ventral posterolateral nuclei of the thalamus. From there they respectively reach the anterior cingulate and anterior insula [44, 49, 50], the latter 2 areas can be source localized with EEG using an inverse solution method such as sLORETA [32]. The ascending lateral pain pathway is activated by $\mathrm{C}, \mathrm{A} \delta$, and $\mathrm{A} \beta$ fibers, and connects to the ventral posterolateral nucleus of the thalamus and from there the pain information is relayed to the somatosensory cortex and parietal area [44, 49]. The descending pain-inhibitory system, also known as the antinociceptive pathway, involves the rostral and pregenual anterior cingulate cortex and connects to the periaqueductal gray and from there to the somatosensory periphery [7, 49], where painful stimuli will be permitted or inhibited to ascend to the brain by activation of the on or off cells [6]. However, the pain-inhibitory pathway also involves other areas such as the periaqueductal gray, the context-processing parahippocampal area [51, 52], anterior insula, hypothalamus, and rostral ventromedial medulla oblongata $[6,7,53]$. The pain-inhibitory pathway is also involved in stress-mediated pain inhibition [54] and placebo analgesia [53], both of which can be considered contextual. Pain perception is, indeed, contextually perceived as unpleasant or sometimes even pleasant. For example, in a very specific, often erotic (sado-masochist) context, pain can be perceived as pleasant, whereas, in general, it is perceived as unpleasant. Undeniably, it has been shown that context changes perception of an identical pain stimulus, and that pleasant pain activates antinociceptive and the reward (accumbens, caudate) system, whereas unpleasant pain activates dorsal anterior cingulate cortex and insula (= salience network) [48]. Thus, the antinociceptive pathway not only suppresses pain, but also increases pleasure. The pregenual anterior cingulate cortex/ventral medial prefrontal cortex, via its connections to the orbitofrontal cortex, also encodes pleasure, irrespective of its cause [55]. In pain particularly, there seems to be a shared emotion appraisal neurocircuitry, which down- or upregulates early sensory processing, depending on whether the expectation is reduced pain or increased pleasure [56]. In fibromyalgia, the antinociceptive pathway is deficient [14], and, more specifically, emotional contextual pain suppression is dysfunctional, even though physiological pain processing is normal [57]. It is thought that the parahippocampal area might encode the contextual [51] aversive memory trace $[58,59]$, and the net effect of context on pain is mediated via modulation of both the medial pain system (i.e., by the dorsal anterior cingulate cortex and insula [57]) and the pain-inhibitory pathway [48].

\section{Pathophysiology of Fibromyalgia}

The results of this study demonstrate that fibromyalgia is associated with increased beta 2 and beta 3 activity in the dorsal anterior cingulate cortex and dorsal lateral prefrontal cortex (Fig. 1, top panel) which are part of the medial pain pathway encoding the motivational/affective component of pain (= unpleasantness) $[20,46,60]$ but no decreased activity in the pregenual anterior cingulate cortex (Fig. 1, top panel). This suggests that fibromyalgia is predominantly related to increased pain sensitization, rather than a decrease in pain inhibition. This leads to an imbalance between pain detection and pain suppression as the pregenual anterior cingulate cortex/dorsal anterior cingulate cortex current density demonstrates (Fig. 1, mid panel). This imbalance between pain input and pain suppression can be simplified by the ratio of source localized current density recorded from the pain-inhibiting 
pregenual anterior cingulate cortex and the current density recorded from the pain-provoking dorsal anterior cingulate cortex. Furthermore, the amount of pain is correlated to the severity of the imbalance as a correlation analysis demonstrates (Fig. 4, lower panel).

If pain is really related to a balance between pain detection (i.e., dorsal anterior cingulate cortex) and pain inhibition (i.e., pregenual anterior cingulate cortex) then these 2 areas need to communicate and it is expected that in a chronic pain condition, such as fibromyalgia, there exists a dysfunction in communication between these 2 areas. This can be evaluated at an intercortical level by functional and effective connectivity measures such as lagged phase coherence and Granger causality (Fig. 5). Results show a definite decreased functional and effective connectivity between the dorsal anterior cingulate cortex and the pregenual anterior cingulate cortex in comparison the healthy controls (Fig. 1, top panel), which demonstrates that fibromyalgia is both an activity and connectivity disorder [61]. Furthermore, the amount of decrease in functional connectivity relates to the amount of pain, pain catastrophizing and impact on life quality perceived by the patients with fibromyalgia, whereas for activity no such correlations could be found (Fig. 5, upper mid-panel). This suggests that the pain and other fibromyalgiarelated symptoms are related to communication between the dACC (salience) and pgACC (pain inhibition). And the amount of reduction of pain and fibromyalgia impact after tDCS correlates with the amount of increase in functional connectivity (Fig. 5, lower mid-panel), demonstrating that these connectivity changes are causally related to the pain. The effective connectivity analysis (Fig. 5, lower panels) further elucidates the underlying mechanism, by showing that in fibromyalgia there is increased dorsal anterior cingulate cortex to pregenual anterior cingulate cortex communication, the amount of which is related to the pain and fibromyalgia impact. We hypothesize that this is an inhibitory pathway, or at least preventing activation of pain inhibition as this is associated with an increase in activation of the dorsal anterior cingulate cortex and not increased pregenual anterior cingulate cortex. The connectivity measures further support the notion that the pain and global perceptual changes in fibromyalgia are related to an imbalance between stimulus detection and stimulus suppression.

In summary, the data suggest that in fibromyalgia, the lack of communication (functional and effective connectivity) is related to the amount of pain perceived by the patients, therefore correlating with an imbalance between pain detection and pain inhibition. Now the question is, how does tDCS of the $\mathrm{C} 2$ dermatoma influence the fibromyalgia-related pain, pain catastrophizing, and subsequent impact on the patients' quality of life?

\section{Occipital Nerve Field tDCS Working Mechanisms}

C2 nerve field stimulation using DC exerts a beneficial effect on pain perception in a placebo-controlled way for pain, pain catastrophizing, and the impact of fibromyalgia on quality of life (Fig. 2), confirming earlier studies [5]. The mechanism by which this is mediated involves activation of the pregenual anterior cingulate cortex/ventral medial prefrontal cortex extending into the ventral lateral prefrontal cortex/dorsal lateral prefrontal cortex and deactivation of the dorsal anterior cingulate cortex mediated through beta oscillations (Fig. 3, lower panel). The occipital nerve field tDCS predominantly activates the pregenual anterior cingulate cortex/ventral medial prefrontal cortex, as seen by the comparison with resting-state activity in healthy volunteers (Fig. 6, top panel).

Furthermore, occipital nerve field stimulation increases the fibromyalgia-related decreased functional connectivity between dorsal anterior cingulate cortex, pregenual anterior cingulate cortex, and the amount of functional connectivity increase correlates with the amount of pain suppression. This suggests there is a causal relationship between the functional connectivity, the perceived pain, and impact of fibromyalgia. The beauty of this technique (tDCS) is that it normalizes functional connectivity, as can be demonstrated by the fact that active tDCS and pain-free healthy controls have the same functional connectivity between pain detection (i.e., dorsal anterior cingulate cortex), pain inhibition (i.e., pregenual anterior cingulate cortex), and normalization of the dorsal anterior cingulate cortex to pregenual anterior cingulate cortex communication. Mechanistically this is mediated through a selective increase in effective connectivity from the pregenual anterior cingulate cortex to the dorsal anterior cingulate cortex, that is, the reverse pattern of the fibromyalgia-related dorsal anterior cingulate cortex to pregenual anterior cingulate cortex communication. In addition, the effective connectivity is normalized. We thus hypothesize that the fibromyalgia-related pain, which is likely due to an inhibitory control of the pain detecting dorsal anterior cingulate cortex on the pain suppressive pregenual anterior cingulate cortex, is reversed by occipital nerve field stimulation, which normalizes the balance between the two areas.

The $\mathrm{C} 2-\mathrm{C} 3$ area in the spinal cord might be a phylogenetically old integration center for combining stimuli from the external environment via somatosensory input and internal environment via the autonomic nervous system. The structures of the brain that regulate the autonomic nervous system consist of the autonomic centers at the brainstem (sympathetic locus coeruleus and parasympathetic nucleus tractus solitarius), as well as supratentorial brain areas involved in autonomic regulation such as the subgenual and dorsal part of the anterior cingulate, the insula, amydala, and the 
hypothalamus. Most of these structures are monosynaptically connected to the neurons at $\mathrm{C} 2$ spinal cord [62]. Furthermore, some neurons in the $\mathrm{C} 2$ spinal cord respond to sympathetic [63] or parasympathetic [64] stimulation, or to both sympathetic and parasympathetic stimulation [63, 65]. Many of the previously mentioned structures are also involved in the sensation of pain $[66,67]$. Furthermore, there are single cells in the myelum at $\mathrm{C} 2$, which respond to the sympathetic, parasympathetic, somatosensory, and phrenic nerve stimulation, suggesting these cells are integrating these different inputs $[63,65]$. Further support for this concept is based on the presence of a somatotopic [68] and viscerotopic [69] organization of the $\mathrm{C} 2-\mathrm{C} 3$ spinal cord. The occipital nerve in the cat, in which the somatotopy has been studied [68], is the main input from the pinna [70]. When a cat hears a sound it turns its ears toward the sound to gain spatial information from where the sound comes. In order to know where the sound is located the cat needs to have a representation of itself in the environment, possibly explaining the reason for the anatomical location of this phylogenetically old integration center. Furthermore, localizing a sound in space is of behavioral importance, thereby possibly explaining why the salience network (pregenual-rostraldorsal anterior cingulate cortex, insula, hypothalamus, amygdala, periaqueductal gray, ventral tegmental area) [71] is monosynaptically connected to the $\mathrm{C} 2$ spinal area [62]. It is of interest that the pregenual anterior cingulate cortex and periaqueductal gray, which are the main areas of the descending pain inhibitory pathway $[6,7]$ (also known as the antinociceptive system) are directly connected to the $\mathrm{C} 2$ area.

A limitation of this study is the low resolution of the source localization inherently resulting from a limited number of EEG sensors (19 electrodes) and a lack of subject-specific anatomic forward models. This is sufficient for source reconstruction but results in greater uncertainty in source localization and decreased anatomical precision, and thus the spatial precision of the present study is considerably lower than that of functional MRI. Nevertheless, sLORETA has received considerable validation from studies combining LORETA with other more established localization methods, such as functional magnetic resonance imaging $[72,73]$, structural magnetic resonance imaging [74], and PET [75-77], and was used in previous studies to detect, for example, activity in the auditory cortex [78-80]. Further sLORETA validation has been based on accepting as ground truth the localization findings obtained from invasive, implanted depth electrodes, in which case there are several studies in epilepsy [81, 82] and cognitive event-related potentials [83]. It is worth emphasizing that deep structures such as the anterior cingulate cortex [84] and mesial temporal lobes [85] can be correctly localized with these methods. However, further research could improve spatial precision and accuracy by using high-density EEG (e.g., 128 or 256 electrodes) and subject-specific head models, and magnetoencephalography recordings. A second limitation is that EEGs and pain scores were only recorded after the final tDCS session and after the final tDCS sham session rather than during all sessions, which would have permitted to analyze the progression of activity and connectivity changes.

\section{Conclusion}

In conclusion, occipital nerve field tDCS seems to have an inhibitory effect on the salience network, which is involved in the affective component/unpleasantness of the pain, as well as an activating effect on the descending pain-inhibitory pathway. It thereby normalizes the imbalance between the painprovoking dorsal anterior cingulate cortex and painsuppressing pregenual anterior cingulate cortex. It can be hypothesized that this effect is predominantly the result of removing the salience attached to the pain (decrease in dorsal anterior cingulate cortex) and improving the perceived nonspecific pleasantness (increase in pregenual anterior cingulate cortex). Mechanistically, this is mediated by inducing changes in effective connectivity, increasing communication from the pain inhibitory pregenual anterior cingulate cortex to the paindetecting dorsal anterior cingulate cortex, and thereby normalizing the fibromyalgia-related abnormal effective connectivity from the hyperactive dorsal anterior cingulate cortex to inhibit the pain suppressive pregenual anterior cingulate cortex. This normalizes functional connectivity between the 2 areas, resulting in a normalization of the imbalance, leading to pain suppression, and therefore improving the impact fibromyalgia has on the patients.

In view of the fact that the dorsal anterior cingulate cortex and pregenual anterior cingulate cortex are nonspecific areas it is tempting to speculate that this mechanism might be involved in multiple pathologies, and is not limited to fibromyalgia. The dorsal anterior cingulate cortex is part of a nonspecific salience network [71], attaching behavioral relevance not only to pain and other somatosensory stimuli, but also to visual and auditory stimuli $[15,19]$. This explains why dorsal anterior cingulate cortex hyperactivity is involved in unpleasantness of pain [46], tinnitus [86], asthmatic dyspnea [87], social phobia [88], and other disorders. Furthermore, the pregenual anterior cingulate cortex seems to be a nonspecific suppressive mechanism suppressing further input of pain- [7], sound- [89], balance- [90], and even aggressiveness- related stimuli [91-93]. This opens up the use of occipital nerve field stimulation in a wide variety of theoretically plausible imbalance disorders, characterized by spontaneous hyperactivity of the salience network clinically expressed as, for example, pain, tinnitus, vertigo, aggression, and other neurological disorders. 
Required Author Forms Disclosure forms provided by the authors are available with the online version of this article.

\section{References}

1. Jenkins, B. and S.J. Tepper, Neurostimulation for primary headache disorders: Part 2, review of central neurostimulators for primary headache, overall therapeutic efficacy, safety, cost, patient selection, and future research in headache neuromodulation. Headache, 2011. 51(9): p. 1408-18.

2. Plazier, M., S. Vanneste, I. Dekelver, M. Thimineur and D. De Ridder, Peripheral nerve stimulation for fibromyalgia. Prog Neurol Surg, 2011. 24: p. 133-46.

3. Thimineur, M. and D. De Ridder, C2 area neurostimulation: a surgical treatment for fibromyalgia. Pain Med, 2007. 8(8): p. 63946.

4. De Ridder, D., M. Plazier, T. Menovsky, N. Kamerling and S. Vanneste, $C 2$ subcutaneous stimulation for failed back surgery syndrome: a case report. Neuromodulation, 2013. 16(6): p. 610-3.

5. Plazier, M., S. Tchen, J. Ost, K. Joos, D. De Ridder and S. Vanneste, Is Transcranial Direct Current Stimulation an Effective Predictor for Invasive Occipital Nerve Stimulation Treatment Success in Fibromyalgia Patients? Neuromodulation, 2015. 18(7): p. 623-9.

6. Fields, H., State-dependent opioid control of pain. Nat Rev Neurosci, 2004. 5(7): p. 565-75.

7. Kong, J., M.L. Loggia, C. Zyloney, P. Tu, P. Laviolette and R.L. Gollub, Exploring the brain in pain: activations, deactivations and their relation. Pain, 2010. 148(2): p. 257-67.

8. Magis, D., M.A. Bruno, A. Fumal, P.Y. Gerardy, R. Hustinx, S. Laureys and J. Schoenen, Central modulation in cluster headache patients treated with occipital nerve stimulation: an FDG-PET study. BMC Neurol, 2011. 11: p. 25.

9. Baliki, M.N., B. Petre, S. Torbey, K.M. Herrmann, L. Huang, T.J. Schnitzer, H.L. Fields and A.V. Apkarian, Corticostriatal functional connectivity predicts transition to chronic back pain. Nat Neurosci, 2012. 15(8): p. 1117-9.

10. Mansour, A.R., M.N. Baliki, L. Huang, S. Torbey, K.M. Herrmann, T.J. Schnitzer and A.V. Apkarian, Brain white matter structural properties predict transition to chronic pain. Pain, 2013. 154(10): p. 2160-8.

11. Wolfe, F. and B. Walitt, Culture, science and the changing nature of fibromyalgia. Nat Rev Rheumatol, 2013. 9(12): p. 751-5.

12. Plazier, M., J. Ost, G. Stassijns, D. De Ridder and S. Vanneste, Pain characteristics in fibromyalgia: understanding the multiple dimensions of pain. Clin Rheumatol, 2015. 34(4): p. 775-83.

13. Cagnie, B., F. Struyf, A. Cools, B. Castelein, L. Danneels and S. O'Leary, The relevance of scapular dysfunction in neck pain: a brief commentary. J Orthop Sports Phys Ther, 2014. 44(6): p. 435-9.

14. Jensen, K.B., P. Srinivasan, R. Spaeth, Y. Tan, E. Kosek, F. Petzke, S. Carville, P. Fransson, H. Marcus, S.C. Williams, E. Choy, O. Vitton, R. Gracely, M. Ingvar and J. Kong, Overlapping structural and functional brain changes in patients with long-term exposure to fibromyalgia pain. Arthritis Rheum, 2013. 65(12): p. 3293-303.

15. Mouraux, A., A. Diukova, M.C. Lee, R.G. Wise and G.D. Iannetti, A multisensory investigation of the functional significance of the "pain matrix". Neuroimage, 2011. 54(3): p. 2237-49.

16. Pujol, J., D. Macia, A. Garcia-Fontanals, L. Blanco-Hinojo, M. Lopez-Sola, S. Garcia-Blanco, V. Poca-Dias, B.J. Harrison, O. Contreras-Rodriguez, J. Monfort, F. Garcia-Fructuoso and J. Deus, The contribution of sensory system functional connectivity reduction to clinical pain in fibromyalgia. Pain, 2014. 155(8): p. 1492-503.
17. Baliki, M.N., P.Y. Geha, A.V. Apkarian and D.R. Chialvo, Beyond feeling: chronic pain hurts the brain, disrupting the default-mode network dynamics. J Neurosci, 2008. 28(6): p. 1398-403.

18. Iannetti, G.D. and A. Mouraux, From the neuromatrix to the pain matrix (and back). Exp Brain Res, 2010. 205(1): p. 1-12.

19. Legrain, V., G.D. Iannetti, L. Plaghki and A. Mouraux, The pain matrix reloaded: a salience detection system for the body. Prog Neurobiol, 2011. 93(1): p. 111-24.

20. De Ridder, D. and S. Vanneste, Burst and Tonic Spinal Cord Stimulation: Different and Common Brain Mechanisms. Neuromodulation, 2016. 19(1): p. 47-59.

21. Plazier, M., I. Dekelver, S. Vanneste, G. Stassijns, T. Menovsky, M. Thimineur and D. De Ridder, Occipital nerve stimulation in fibromyalgia: a double-blind placebo-controlled pilot study with a sixmonth follow-up. Neuromodulation, 2014. 17(3): p. 256-64.

22. Plazier, M., J. Ost, G. Stassijns, D. De Ridder and S. Vanneste, $C 2$ Nerve Field Stimulation for the Treatment of Fibromyalgia: A Prospective, Double-blind, Randomized, Controlled Cross-over Study. Brain Stimul, 2015. 8(4): p. 751-7.

23. Vanneste, S., B. Langguth and D. De Ridder, Do tDCS and TMS influence tinnitus transiently via a direct cortical and indirect somatosensory modulating effect? A combined TMS-tDCS and TENS study. Brain Stimul, 2011. 4(4): p. 242-52.

24. Plazier, M., J. Ost, E. Snijders, M. Gilbers, T. Vancamp, D. De Ridder and S. Vanneste, Laser-Evoked Potentials in Fibromyalgia: The Influence of Greater Occipital Nerve Stimulation on Cerebral Pain Processing. Neuromodulation, 2015. 18(5): p. 376-83.

25. Kovacs, S., R. Peeters, D. De Ridder, M. Plazier, T. Menovsky and S. Sunaert, Central effects of occipital nerve electrical stimulation studied by functional magnetic resonance imaging. Neuromodulation, 2011. 14(1): p. 46-55; discussion 56-7.

26. Garcia-Larrea, L. and R. Peyron, Pain matrices and neuropathic pain matrices: a review. Pain, 2013. 154 Suppl 1: p. S29-43.

27. Wolfe, F., H.A. Smythe, M.B. Yunus, R.M. Bennett, C. Bombardier, D.L. Goldenberg, P. Tugwell, S.M. Campbell, M. Abeles, P. Clark and et al., The American College of Rheumatology 1990 Criteria for the Classification of Fibromyalgia. Report of the Multicenter Criteria Committee. Arthritis Rheum, 1990. 33(2): p. 160-72.

28. Plazier, M., J. Ost, G. Stassijns, D. De Ridder and S. Vanneste, Pain characteristics in fibromyalgia: understanding the multiple dimensions of pain. Clin Rheumatol, 2014.

29. Bennett, R., The Fibromyalgia Impact Questionnaire (FIQ): a review of its development, current version, operating characteristics and uses. Clin Exp Rheumatol, 2005. 23(5 Suppl 39): p. S154-62.

30. Osman, A., F.X. Barrios, B.A. Kopper, W. Hauptmann, J. Jones and E. O'Neill, Factor structure, reliability, and validity of the Pain Catastrophizing Scale. J Behav Med, 1997. 20(6): p. 589-605.

31. Congedo, M., EureKa! (Version 3.0) [Computer Software]. Knoxville, TN: NovaTech EEG Inc. Freeware available at www. NovaTechEEG. 2002.

32. Pascual-Marqui, R.D., Standardized low-resolution brain electromagnetic tomography (sLORETA): technical details. Methods Find Exp Clin Pharmacol, 2002. 24 Suppl D: p. 5-12.

33. Jurcak V, Tsuzuki D, Dan I. 10/20, 10/10, and 10/5 systems revisited: their validity as relative headsurface-based positioning systems. Neuroimage. Feb 15 2007. 34(4): p.1600-1611.

34. Fuchs A. Combining Brain Imaging Technologies: Using Brain Surfaces. In: H. Nowak, J. Haueisen, F. Geißler, Huonker R, eds. Biomag 2002, Proceedings of the 13th International Conference on Biomagnetism. Berlin: VDE Verlag; 2002. p. 878-880.

35. Lancaster JL, Woldorff MG, Parsons LM, et al. Automated Talairach atlas labels for functional brain mapping. Hum Brain Mapp. Jul 2000. 10(3): p. 120-131. 
36. Pascual-Marqui, R. Instantaneous and lagged measurements of linear and nonlinear dependence between groups of multivariate time series: frequency decomposition. 2007. https://arxiv. org/ftp/arxiv/papers/0711/0711.1455.pdf

37. Pascual-Marqui, R. Discrete, $3 D$ distributed, linear imaging methods of electric neuronal activity. Part 1: exact, zero error localization. 2007. https://arxiv.org/abs/0710.3341

38. Congedo, M., R.E. John, D. De Ridder, L. Prichep and R. Isenhart, On the "dependence" of "independent" group EEG sources; an EEG study on two large databases. Brain Topogr, 2010. 23(2): p. 134-8.

39. Bloomfield P. Fourier Analysis of Time Series: An Introduction, 2nd Edition. New York: Wiley; 2000.

40. Geweke, J., Measurement of lineair dependence and feedback between multiple time series. J. Am. Stat. Assoc., 1982. 77: p. 304.

41. Granger, C.W.J., Investigating causal relations by econometrics models and crosss-spectral methods. Econometrica, 1969. 37: p. 424.

42. Nichols, T.E. and A.P. Holmes, Nonparametric permutation tests for functional neuroimaging: a primer with examples. Hum Brain Mapp, 2002. 15(1): p. 1-25.

43. Frank, E., S. Wilfurth, M. Landgrebe, P. Eichhammer, G. Hajak and B. Langguth, Anodal skin lesions after treatment with transcranial direct current stimulation. Brain Stimul, 2010. 3(1): p. 58-9.

44. Price, D.D., Psychological and neural mechanisms of the affective dimension of pain. Science, 2000. 288(5472): p. 1769-72.

45. Bushnell, M.C., M. Ceko and L.A. Low, Cognitive and emotional control of pain and its disruption in chronic pain. Nat Rev Neurosci, 2013. 14(7): p. 502-11.

46. Rainville, P., G.H. Duncan, D.D. Price, B. Carrier and M.C. Bushnell, Pain affect encoded in human anterior cingulate but not somatosensory cortex. Science, 1997. 277(5328): p. 968-71.

47. Frot, M., F. Mauguiere, M. Magnin and L. Garcia-Larrea, Parallel processing of nociceptive A-delta inputs in SII and midcingulate cortex in humans. J Neurosci, 2008. 28(4): p. 944-52.

48. Leknes, S., C. Berna, M.C. Lee, G.D. Snyder, G. Biele and I. Tracey, The importance of context: when relative relief renders pain pleasant. Pain, 2013. 154(3): p. 402-10.

49. Craig, A.D., How do you feel? Interoception: the sense of the physiological condition of the body. Nat Rev Neurosci, 2002. 3(8): p. 655-66.

50. Craig, A.D., Distribution of trigeminothalamic and spinothalamic lamina I terminations in the macaque monkey. J Comp Neurol, 2004. 477(2): p. 119-48.

51. Aminoff, E., N. Gronau and M. Bar, The parahippocampal cortex mediates spatial and nonspatial associations. Cereb Cortex, 2007. 17(7): p. 1493-503.

52. Aminoff, E.M., K. Kveraga and M. Bar, The role of the parahippocampal cortex in cognition. Trends Cogn Sci, 2013. 17(8): p. 379-90.

53. Eippert, F., U. Bingel, E.D. Schoell, J. Yacubian, R. Klinger, J. Lorenz and C. Buchel, Activation of the opioidergic descending pain control system underlies placebo analgesia. Neuron, 2009. 63(4): p. 533-43

54. Yilmaz, P., M. Diers, S. Diener, M. Rance, M. Wessa and H. Flor, Brain correlates of stress-induced analgesia. Pain, 2010. 151(2): $\mathrm{p}$. 522-9.

55. Kuhn, S. and J. Gallinat, The neural correlates of subjective pleasantness. Neuroimage, 2012. 61(1): p. 289-94.

56. Ellingsen, D.M., J. Wessberg, M. Eikemo, J. Liljencrantz, T. Endestad, H. Olausson and S. Leknes, Placebo improves pleasure and pain through opposite modulation of sensory processing. Proc Natl Acad Sci U S A, 2013. 110(44): p. 17993-8.

57. Kamping, S., I.C. Bomba, P. Kanske, E. Diesch and H. Flor, Deficient modulation of pain by a positive emotional context in fibromyalgia patients. Pain, 2013. 154(9): p. 1846-55.
58. De Ridder, D., A.B. Elgoyhen, R. Romo and B. Langguth, Phantom percepts: tinnitus and pain as persisting aversive memory networks. Proc Natl Acad Sci U S A, 2011. 108(20): p. 8075-80.

59. Flor, H., L. Nikolajsen and T. Staehelin Jensen, Phantom limb pain: a case of maladaptive CNS plasticity? Nat Rev Neurosci, 2006. 7(11): p. 873-81.

60. De Ridder, D., M. Plazier, N. Kamerling, T. Menovsky and S. Vanneste, Burst spinal cord stimulation for limb and back pain. World Neurosurg, 2013. 80(5): p. 642-649 e1.

61. Fornito, A. and E.T. Bullmore, Connectomics: A new paradigm for understanding brain disease. Eur Neuropsychopharmacol, 2015. 25(5): p. 733-48.

62. Newman, H.M., R.T. Stevens and A.V. Apkarian, Direct spinal projections to limbic and striatal areas: anterograde transport studies from the upper cervical spinal cord and the cervical enlargement in squirrel monkey and rat. J Comp Neurol, 1996. 365(4): p. 640-58.

63. Chandler, M.J., J. Zhang and R.D. Foreman, Vagal, sympathetic and somatic sensory inputs to upper cervical (C1-C3) spinothalamic tract neurons in monkeys. J Neurophysiol, 1996. 76(4): p. 2555-67.

64. Chandler, M.J., S.F. Hobbs, D.C. Bolser and R.D. Foreman, Effects of vagal afferent stimulation on cervical spinothalamic tract neurons in monkeys. Pain, 1991. 44(1): p. 81-7.

65. Chandler, M.J., C. Qin, Y. Yuan and R.D. Foreman, Convergence of trigeminal input with visceral and phrenic inputs on primate C1-C2 spinothalamic tract neurons. Brain Res, 1999. 829(1-2): p. 204-8.

66. Leone, M., A. Proietti Cecchini, E. Mea, V. Tullo, M. Curone and G. Bussone, Neuroimaging and pain: a window on the autonomic nervous system. Neurol Sci, 2006. 27 Suppl 2: p. S134-7.

67. Boly, M., E. Balteau, C. Schnakers, C. Degueldre, G. Moonen, A. Luxen, C. Phillips, P. Peigneux, P. Maquet and S. Laureys, Baseline brain activity fluctuations predict somatosensory perception in humans. Proc Natl Acad Sci U S A, 2007. 104(29): p. 12187-92.

68. Smith, M.V., A.V. Apkarian and C.J. Hodge, Jr., Somatosensory response properties of contralaterally projecting spinothalamic and nonspinothalamic neurons in the second cervical segment of the cat. J Neurophysiol, 1991. 66(1): p. 83-102.

69. Kanold, P.O. and E.D. Young, Proprioceptive information from the pinna provides somatosensory input to cat dorsal cochlear nucleus. $\mathrm{J}$ Neurosci, 2001. 21(19): p. 7848-58.

70. Kanold, P.O. and E.D. Young, Proprioceptive information from the pinna provides somatosensory input to cat dorsal cochlear nucleus. $\mathbf{J}$ Neurosci 2001.21(19): p. 7848-7858.

71. Seeley, W.W., V. Menon, A.F. Schatzberg, J. Keller, G.H. Glover, H. Kenna, A.L. Reiss and M.D. Greicius, Dissociable intrinsic connectivity networks for salience processing and executive control. J Neurosci, 2007. 27(9): p. 2349-56.

72. Mulert, C., L. Jager, R. Schmitt, P. Bussfeld, O. Pogarell, H.J. Moller, G. Juckel and U. Hegerl, Integration of fMRI and simultaneous EEG: towards a comprehensive understanding of localization and timecourse of brain activity in target detection. Neuroimage, 2004. 22(1): p. 83-94.

73. Vitacco, D., D. Brandeis, R. Pascual-Marqui and E. Martin, Correspondence of event-related potential tomography and functional magnetic resonance imaging during language processing. Hum Brain Mapp, 2002. 17(1): p. 4-12.

74. Worrell, G.A., T.D. Lagerlund, F.W. Sharbrough, B.H. Brinkmann, N.E. Busacker, K.M. Cicora and T.J. O'Brien, Localization of the epileptic focus by low-resolution electromagnetic tomography in patients with a lesion demonstrated by MRI. Brain Topogr, 2000. 12(4): p. 273-82.

75. Dierks, T., V. Jelic, R.D. Pascual-Marqui, L. Wahlund, P. Julin, D.E. Linden, K. Maurer, B. Winblad and A. Nordberg, Spatial pattern of cerebral glucose metabolism (PET) correlates with localization of 
intracerebral EEG-generators in Alzheimer's disease. Clin Neurophysiol, 2000. 111(10): p. 1817-24.

76. Pizzagalli, D.A., T.R. Oakes, A.S. Fox, M.K. Chung, C.L. Larson, H.C. Abercrombie, S.M. Schaefer, R.M. Benca and R.J. Davidson, Functional but not structural subgenual prefrontal cortex abnormalities in melancholia. Mol Psychiatry, 2004. 9(4): p. 325, 393-405.

77. Zumsteg, D., R.A. Wennberg, V. Treyer, A. Buck and H.G. Wieser, H2(15)O or 13 NH3 PET and electromagnetic tomography (LORETA) during partial status epilepticus. Neurology, 2005. 65(10): p. 1657-60.

78. Zaehle,T.,L.JanckeandM.Meyer,Electricalbrainimagingevidences leftauditorycortexinvolvementinspeech andnon-speechdiscrimination based on temporalfeatures. Behav Brain Funct,2007.3:p.63.

79. Vanneste, S., M. Plazier, E. van der Loo, P. Van de Heyning and D. De Ridder, The difference between uni- and bilateral auditory phantom percept. Clin Neurophysiol, 2010.

80. Vanneste, S., M. Plazier, E. van der Loo, P. Van de Heyning and D. De Ridder, The difference between uni- and bilateral auditory phantom percept. Clin Neurophysiol, 2011. 122(3): p. 578-87.

81. Zumsteg, D., A.M. Lozano and R.A. Wennberg, Depth electrode recorded cerebral responses with deep brain stimulation of the anterior thalamus for epilepsy. Clin Neurophysiol, 2006. 117(7): p. 1602-9.

82. Zumsteg, D., A.M. Lozano, H.G. Wieser and R.A. Wennberg, Cortical activation with deep brain stimulation of the anterior thalamus for epilepsy. Clin Neurophysiol, 2006. 117(1): p. 192-207.

83. Volpe, U., A. Mucci, P. Bucci, E. Merlotti, S. Galderisi and M. Maj, The cortical generators of P3a and P3b: a LORETA study. Brain Res Bull, 2007. 73(4-6): p. 220-30.

84. Pizzagalli, D., R.D. Pascual-Marqui, J.B. Nitschke, T.R. Oakes, C.L. Larson, H.C. Abercrombie, S.M. Schaefer, J.V. Koger, R.M. Benca and R.J.Davidson, Anterior cingulate activity as a predictor of degree of treatment response in major depression: evidence from brain electrical tomography analysis. Am J Psychiatry, 2001. 158(3): p. 405-15.

85. Zumsteg, D., A.M. Lozano and R.A. Wennberg, Mesial temporal inhibition in a patient with deep brain stimulation of the anterior thalamus for epilepsy. Epilepsia, 2006. 47(11): p. 1958-62.

86. Vanneste, S., M.Plazier, E. derLoo, P.V. deHeyning, M.Congedo and D. De Ridder, The neural correlates of tinnitus-related distress. Neuroimage, 2010. 52(2): p. 470-80.

87. von Leupoldt, A., T. Sommer, S. Kegat, H.J. Baumann, H. Klose, B. Dahme and C. Buchel, Dyspnea and pain share emotion-related brain network. Neuroimage, 2009. 48(1): p. 200-6.

88. Mataix-Cols, D., S.K. An, N.S. Lawrence, X. Caseras, A. Speckens, V. Giampietro, M.J. Brammer and M.L. Phillips, Individual differences in disgust sensitivity modulate neural responses to aversivel disgusting stimuli. Eur J Neurosci, 2008. 27(11): p. 3050-8.

89. Song, J.J., S. Vanneste and D. De Ridder, Dysfunctional noise cancelling of the rostral anterior cingulate cortex in tinnitus patients. PLoS One, 2015. 10(4): p. e0123538.

90. Alsalman, O., J. Ost, R. Vanspauwen, C. Blaivie, D. De Ridder and S. Vanneste, The Neural Correlates of Chronic Symptoms of Vertigo Proneness in Humans. PLoS One, 2016. 11(4): p. e0152309.

91. Buckholtz, J.W. and A. Meyer-Lindenberg, MAOA and the neurogenetic architecture of human aggression. Trends Neurosci, 2008. 31(3): p. 120-9.

92. Eisenberger, N.I., B.M. Way, S.E. Taylor, W.T. Welch and M.D. Lieberman, Understanding genetic risk for aggression: clues from the brain's response to social exclusion. Biol Psychiatry, 2007. 61(9): p. 1100-8.

93. Meyer-Lindenberg, A., J.W. Buckholtz, B. Kolachana, R.H. A, L. Pezawas, G. Blasi, A. Wabnitz, R. Honea, B. Verchinski, J.H. Callicott, M. Egan, V. Mattay and D.R. Weinberger, Neural mechanisms of genetic risk for impulsivity and violence in humans. Proc Natl Acad Sci U S A, 2006. 103(16): p. 6269-74. 